\title{
Gut dendritic cell activation links an altered colonic microbiome to mucosal and systemic T-cell activation in untreated HIV-1 infection
}

\author{
SM Dillon ${ }^{1}$, EJ Lee ${ }^{1}$, CV Kotter ${ }^{1}$, GL Austin ${ }^{2}$, S Gianella ${ }^{3}$, B Siewe ${ }^{4}$, DM Smith ${ }^{3}$, AL Landay ${ }^{4}$, \\ MC McManus ${ }^{5}$, CE Robertson ${ }^{1,6}$, DN Frank ${ }^{1,6}$, MD McCarter ${ }^{7}$ and CC Wilson ${ }^{1}$
}

HIV-1-associated disruption of intestinal homeostasis is a major factor contributing to chronic immune activation and inflammation. Dendritic cells (DCs) are crucial in maintaining intestinal homeostasis, but the impact of HIV-1 infection on intestinal DC number and function has not been extensively studied. We compared the frequency and activation/ maturation status of colonic myeloid $\mathrm{DC}(\mathrm{mDC})$ subsets $\left(\mathrm{CD} 1 \mathrm{c}^{+}\right.$and $\left.\mathrm{CD1} \mathrm{c}^{\text {neg }}\right)$ and plasmacytoid $\mathrm{DCs}$ in untreated HIV-1-infected subjects with uninfected controls. Colonic mDCs in HIV-1-infected subjects had increased CD40 but decreased CD83 expression, and CD40 expression on CD1c ${ }^{+}$mDCs positively correlated with mucosal HIV-1 viral load, with mucosal and systemic cytokine production, and with frequencies of activated colon and blood Tcells. Percentage of $\mathrm{CD}_{3} 3^{+} \mathrm{CD}_{1 \mathrm{c}}{ }^{+} \mathrm{mDC}$ negatively correlated with frequencies of interferon- $\gamma$-producing colon CD4 ${ }^{+}$and CD8 ${ }^{+} \mathrm{Tcells}$ CD40 expression on $\mathrm{CD} \mathrm{c}^{+} \mathrm{mDCs}$ positively associated with abundance of high prevalence mucosal Prevotella copri and Prevotella stercorea but negatively associated with a number of low prevalence mucosal species, including Rumminococcus bromii. $\mathrm{CD} 1 \mathrm{c}^{+} \mathrm{mDC}$ cytokine production was greater in response to in vitro stimulation with Prevotella species relative to $R$. bromii. These findings suggest that, during HIV infection, colonic mDCs become activated upon exposure to mucosal pathobiont bacteria leading to mucosal and systemic immune activation.

\section{INTRODUCTION}

A hallmark of HIV-1 disease is a gradual decline in peripheral blood CD4 T cells associated with chronic immune activation, defined by increased levels of pro-inflammatory cytokines, innate and adaptive immune cell activation, and soluble markers of inflammation. ${ }^{1}$ T-cell activation, and in particular $\mathrm{CD}^{+}$T-cell activation, has been shown to be a strong predictor of disease progression. ${ }^{2-4}$ In the era of combination anti-retroviral therapy (cART), low levels of T-cell activation and inflammation persist in many individuals despite controlled viral replication and have been linked to poor immune reconstitution and adverse clinical outcomes. ${ }^{1}$ Thus understanding the mechanisms that drive chronic immune activation and the attendant inflammation in the setting of HIV-1 infection is important in order to develop therapeutic approaches to prevent inflammation-associated morbidity and mortality.

Although multiple factors likely contribute to chronic immune activation during HIV-1 infection, microbial translocation (MT) - the movement of bacteria or bacteria products from the gut lumen into the lamina propria (LP) and systemic circulation-has recently been implicated as a major driving force. ${ }^{5}$ Plasma bacterial lipopolysaccharide (LPS) levels have been associated with systemic T-cell activation, and LPS levels in the first years of chronic HIV-1 infection were found to predict HIV-1 disease progression. ${ }^{6,7}$ In addition to LPS, other indicators of systemic MT such as sCD14, intestinal fatty acidbinding protein, and zonulin have also been associated with

\footnotetext{
${ }^{1}$ Department of Medicine, University of Colorado Anschutz Medical Campus, Aurora, Colorado, USA. ${ }^{2}$ Department of Gastroenterology, University of Colorado Anschutz Medical Campus, Aurora, Colorado, USA. ${ }^{3}$ Division of Infectious Diseases, University of California San Diego, La Jolla, California, USA. ${ }^{4}$ Department of ImmunologyMicrobiology, Rush University Medical Center, Chicago, Illinois, USA. ${ }^{5}$ Department of Pathology, University of Colorado Anschutz Medical Campus, Aurora, Colorado, USA. ${ }^{6}$ University of Colorado Microbiome Research Consortium, Aurora, Colorado, USA and ${ }^{7}$ Department of Surgery, University of Colorado Anschutz Medical Campus, Aurora, Colorado, USA. Correspondence: CC Wilson (cara.wilson@ucdenver.edu)
} 
disease progression in untreated and with mortality in treated, HIV-1-infected subjects. ${ }^{8,9}$

Increased MT occurs as a result of HIV-1-associated immunological and structural damage to the gastrointestinal tract. Within days of infection, irrespective of the route of transmission, HIV-1 replication results in severe and rapid depletion of intestinal memory CD4 T cells, including preferential depletion of $\mathrm{T}$ helper type 17 (Th17) and Th22 cells, T-cell subsets involved in normal mucosal defense, and epithelial barrier maintenance. ${ }^{10}$ In addition, increased activated $\mathrm{CD} 8{ }^{+}$T-cell frequencies, ${ }^{11-13}$ increased pro-inflammatory cytokines, ${ }^{14}$ and alterations in the composition of microbial communities have been observed in the gastrointestinal tract of HIV-1-infected subjects. ${ }^{10,15}$ We recently identified an altered colonic mucosal microbiome in untreated, HIV-infected subjects that was associated with plasma LPS levels and mucosal and systemic T-cell activation. ${ }^{16}$ Furthermore, these altered microbial communities were associated with increased expression of the activation marker CD40 on intestinal myeloid dendritic cells (mDCs).

Intestinal DCs sample luminal microbes and their products and are critical in mediating the delicate balance between immunogenic and tolerogenic intestinal immune responses, ${ }^{17}$ yet few studies have directly addressed the contribution of intestinal DCs to HIV-1-associated mucosal pathogenesis. We previously identified a subset of resident $\mathrm{mDC}$ present in the LP of normal small and large bowel that were capable of producing pro-inflammatory cytokines (including interleukin (IL)-23) in response to in vitro stimulation with a viral Toll-like receptor (TLR) ligand that mimicked innate signaling by HIV-1. ${ }^{18}$ Moreover, levels of pro-inflammatory IL-23 were synergistically increased when $\mathrm{mDC}$ were stimulated by a combination of bacterial and viral TLR ligands, suggesting that during HIV-1 infection concurrent exposure to both virus and translocating enteric bacteria and bacterial products could result in enhanced production of pro-inflammatory cytokines by intestinal mDCs in vivo. Further, we showed that exposure to certain commensal bacteria enhanced HIV-1 infection of intestinal CD4 T cells in vitro, and this process was dependent on the presence of mDCs. ${ }^{19}$ Based on these findings and the likelihood that LP DCs would be exposed to translocating mucosa-associated bacteria, we hypothesized that intestinal DCs would have a critical role in mediating viral and bacterial signals during HIV-1 infection in vivo.

\section{RESULTS}

CD40 expression is increased and CD83 expression decreased on colonic mDCs in HIV-1-infected subjects

Twenty-four HIV-1-infected individuals and 14 age- and sexmatched HIV-1 uninfected controls were enrolled into a crosssectional study from whom rectosigmoid biopsies, peripheral blood, and stool samples were collected. Based on study entry criteria, HIV-1-infected subjects were ART-treatment naive or had not been on treatment for $>7$ days in the preceding 6 months. Of the $24 \mathrm{HIV}$-1-infected subjects, 5 subjects reported in a study questionnaire that they had taken ART at some point during their course of HIV-1 infection. Of these, three had stopped ART at least 8 years (range $8-14$ years) prior to the study, one subject stopped 3 years prior, and the remaining subject stopped 13 months prior to inclusion in our study. Additional exclusion criteria are detailed in Supplementary Materials and Methods online. Subject characteristics are provided in Table 1.

In initial studies, two phenotypically distinct colonic LP $\mathrm{mDC}$ subsets were identified, both of which expressed HLA-DR

Table 1 Subject characteristics

\begin{tabular}{|c|c|c|}
\hline & Uninfected subjects & HIV-1-infected subjects \\
\hline Age (years) & $31(23-54)$ & $33.5(22-58)$ \\
\hline CD4 count (cells $\left.\mu\right|^{-1}$ ) & $724(468-1071)$ & $445(221-1248)^{\star}$ \\
\hline Plasma viral load (HIV-1 RNA copies $\mathrm{ml}^{-1}$ ) & - & $51350(2880-207000)$ \\
\hline \multicolumn{3}{|l|}{ Ethnicity } \\
\hline Non-Hispanic & 11 (78.6\%) & 19 (79.2\%) \\
\hline Hispanic & $3(21.4 \%)$ & $5(20.8 \%)$ \\
\hline Asian & $2(14.2 \%)$ & $1(4.2 \%)$ \\
\hline
\end{tabular}

Values are shown as median (range) except for ethnicity and race, which are shown as the number and percentage of each cohort. Statistical analysis was performed using Mann-Whitney test for comparisons between uninfected and HIV-1-infected subjects and Fisher's exact test or Chi-square test for comparison of categorical data. ${ }^{*} P=0.001$. 
and $\mathrm{CD} 11 \mathrm{c}$ but were delineated by the expression of CD1c (Supplementary Results, Supplementary Figure S1). Similar frequencies of both $\mathrm{CD} 1 \mathrm{c}^{+}$and $\mathrm{CD} 1 \mathrm{c}^{\text {neg }} \mathrm{mDCs}$ were observed in uninfected and HIV-1-infected subjects when enumerated as either a percentage of viable, $\mathrm{CD} 45^{+}$cells (Supplementary Table S1) or as an absolute number of DCs per g of mucosal tissue (Figure 1a). Histological techniques were also utilized to enumerate $\mathrm{CD} 11 \mathrm{c}^{+} \mathrm{DCs}$ and HAM $56^{+}$tissue macrophages in colonic tissue sections obtained from a subset of HIV-1infected $(n=6)$ and uninfected $(n=6)$ subjects. A similar number of $\mathrm{CD} 11 \mathrm{c}^{+}$DCs were enumerated in both cohorts (HIV-1-infected: median $12.1 \mathrm{CD} 11 \mathrm{c}^{+}$cells $\mathrm{mm}^{-2}$, range 3.8-

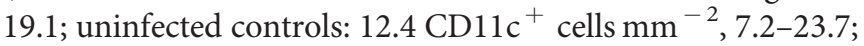
$P=0.75)$, but a higher number of HAM56 ${ }^{+}$cells per $\mathrm{mm}^{2}$ of tissue were found in HIV-1-infected subjects (15.7 HAM56 ${ }^{+}$ cells $\left.\mathrm{mm}^{-2}, 8.7-20.3\right)$ compared with uninfected controls (3.9 ${\text { HAM } 56^{+} \text {cells mm }}^{-2}, 2.3-13.0 ; P=0.02$ ). Increased frequencies of macrophages have also recently been reported in the duodenal mucosa of treatment-naive HIV-1-infected subjects. ${ }^{20}$

Colon $\mathrm{CD} 33^{+}$pDCs, normally found at very low frequencies, ${ }^{18}$ were next assessed for frequency and activation status. We did not observe any statistical difference in the frequencies of colonic pDCs (Figure 1a, Supplementary Table S1) although a trend toward higher numbers of pDCs in HIV-1infected subjects (median: 9354 pDC per g, 1835-59658) compared with uninfected subjects (4243, 2104-14155; $P=0.09)$ was noted.
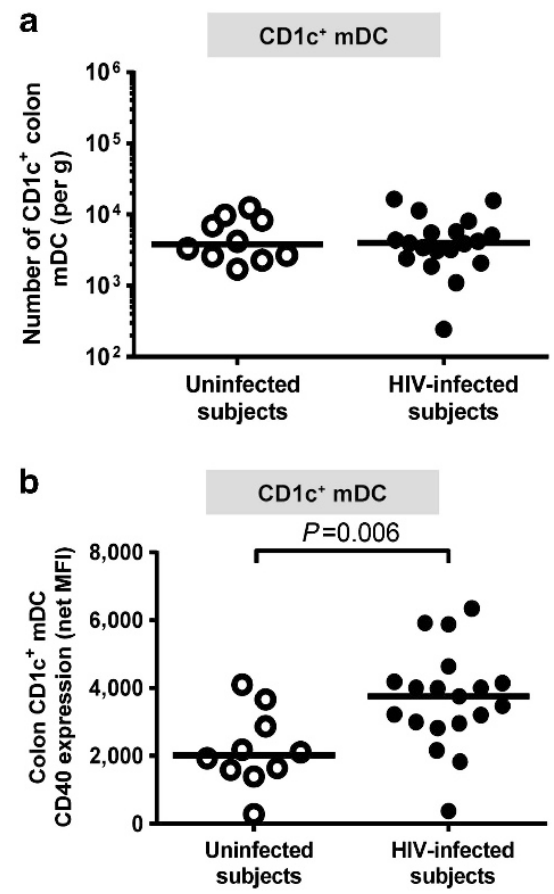

c

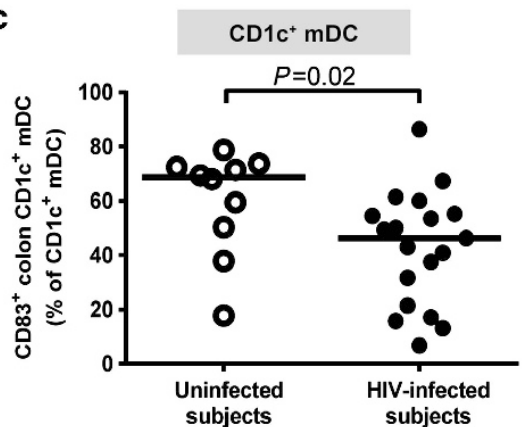

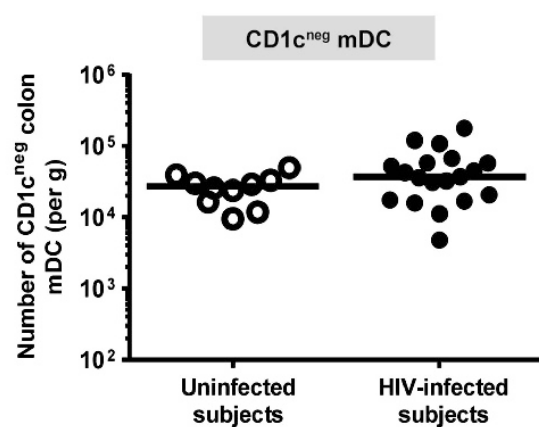
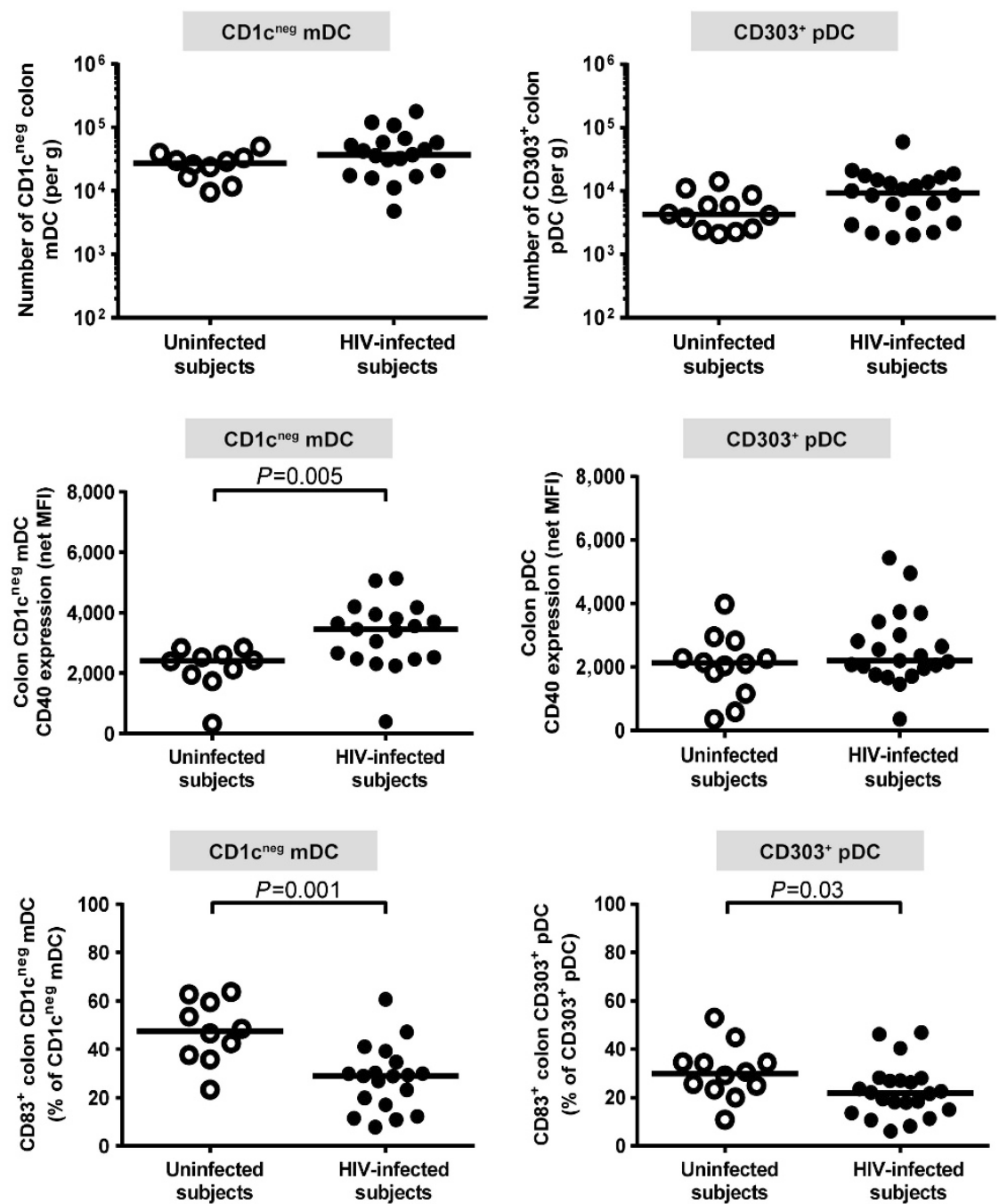

Figure 1 Colon dendritic cells (DCs) from HIV-1-infected subjects have an altered activation profile. Multi-color flow cytometry techniques were used to determine frequencies and activation/maturation states of colon CD1c ${ }^{+}$myeloid DCs $(\mathrm{mDCs}), \mathrm{CD} 1 \mathrm{c}^{\text {neg }} \mathrm{mDCs}$ and CD303 ${ }^{+}$plasmacytoid DCs $(\mathrm{pDCs})$ in uninfected (open circles) and HIV-1-infected (HIV-infected; closed circles) subjects. (a) Frequencies of CD1c ${ }^{+}$mDCs, CD1c ${ }^{\text {neg }}$ mDCs (uninfected $n=10$; HIV-infected $n=19$ ) and $\mathrm{CD}_{30}{ }^{+} \operatorname{pDCs}$ (uninfected $n=12$, HIV-infected $n=22$ ) were evaluated as a percentage of viable, CD45 ${ }^{+}$leucocytes and converted into a total number of DC per g of tissue. (b) CD40 expression levels (mean fluorescence intensity (MFI)) and (c) percentage of CD83 ${ }^{+}$ DCs were assessed on CD1c ${ }^{+}$mDCs, CD1c ${ }^{\text {neg }}$ mDCs (uninfected $n=10 ;$ HIV-infected $n=19$ ), and CD303 ${ }^{+}$pDCs (uninfected $n=12$, HIV-infected $n=22$ ). Appropriate isotype controls were removed to control for background staining (net). Lines represent median values and statistical analysis was performed using Mann-Whitney test. 


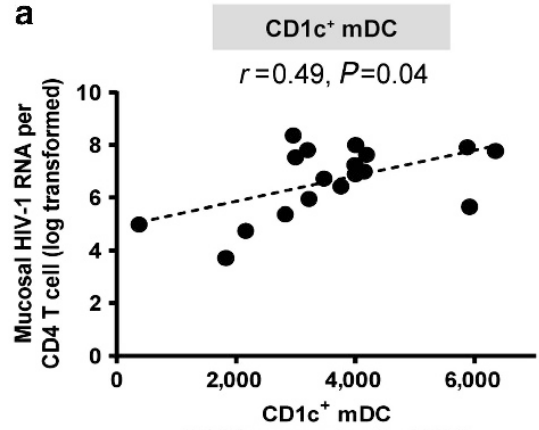

CD40 expression (net MFI)

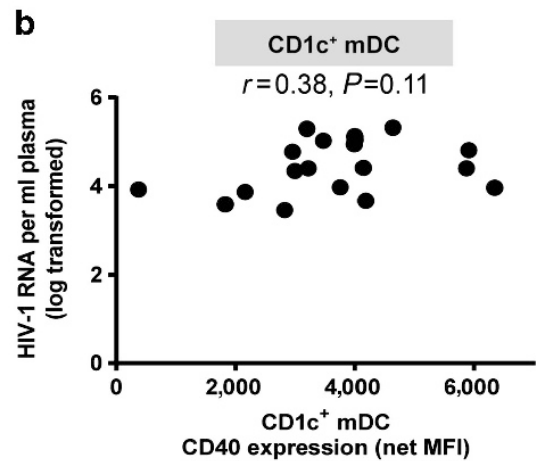

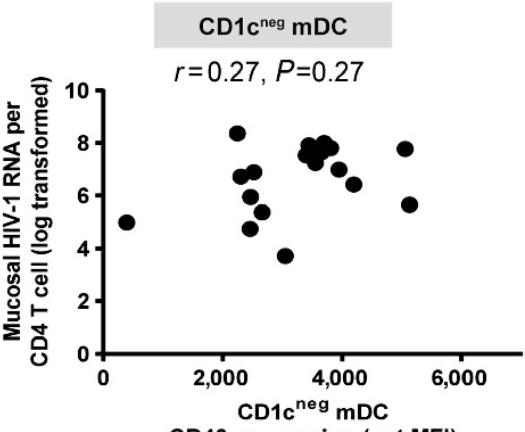

CD40 expression (net MFI)

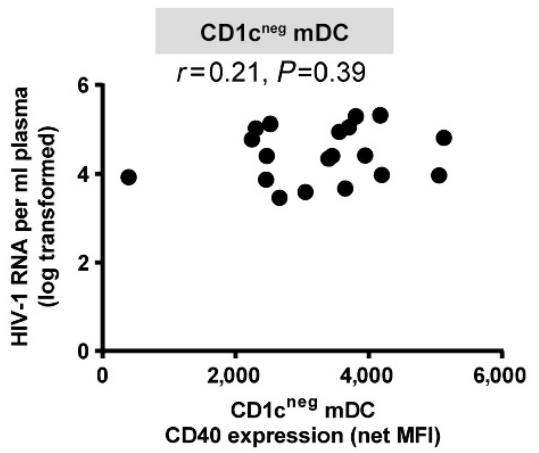

Figure 2 Activated colon $\mathrm{CD}_{1}{ }^{+}$myeloid dendritic cells (mDCs) correlate with mucosal HIV-1 viral load. Correlations between CD40 expression levels (mean fluorescence intensity (MFI)) on CD1c ${ }^{+}$and CD1c ${ }^{\text {neg }} \mathrm{mDCs}$ (shown with background isotype values removed; net MFI) with (a) mucosal HIV-1 viral load $(n=18)$ and $(\mathbf{b})$ plasma HIV-1 viral load $(n=19)$. Statistical analysis was performed using Spearman's test. Dotted line is a visual representation of the significant association.

Colonic $\mathrm{CD} 1 \mathrm{c}^{+} \mathrm{mDC}$ and $\mathrm{CD} 1 \mathrm{c}^{\text {neg }} \mathrm{mDC}$ activation based on CD40 expression was significantly higher in HIV-1-infected subjects compared with uninfected controls (Figure 1b). Conversely, CD40 expression on pDC was not statistically different between the two subject cohorts (Figure 1b). However, the absolute number of $\mathrm{CD}_{40}{ }^{+}$pDCs was statistically greater in HIV-1-infected subjects $\left(9047 \mathrm{CD} 40^{+}\right.$pDC per g, 561-56192; $n=21$ ) compared with uninfected subjects (4380, 2212-11500; $n=21 ; P<0.05)$. CD1c ${ }^{+} \mathrm{mDC}$ activation levels significantly correlated with the number of $\mathrm{CD} 40^{+}$ colonic pDCs ( $r=0.61, P=0.007 ; n=18)$. The percentage of $\mathrm{CD} 1 \mathrm{c}^{+} \mathrm{mDCs}, \mathrm{CD} 1 \mathrm{c}^{\text {neg }} \mathrm{mDCs}$, and $\mathrm{CD} 303^{+}$pDCs expressing the DC maturation marker CD83 were all lower in HIV-1infected subjects (Figure 1c).

\section{Colonic $\mathrm{CD} 1 \mathrm{c}^{+} \mathrm{mDC}$ activation is associated with mucosal} HIV-1 viral load

$\mathrm{CD} 40$ expression on $\mathrm{CD}^{+} \mathrm{c}^{+} \mathrm{mDCs}$ positively associated with mucosal HIV-1 viral load, whereas $\mathrm{CD} 1 \mathrm{c}^{\text {neg }} \mathrm{mDC}$ CD40 expression did not (Figure 2a). Unlike our previous observations of activated blood DCs, ${ }^{21}$ CD40 expression on colon $\mathrm{CD} 1 \mathrm{c}^{+} \mathrm{mDCs}$ and $\mathrm{CD} 1 \mathrm{c}^{\text {neg }} \mathrm{mDC}$ did not correlate with either plasma viral load (Figure $\mathbf{2 b}$ ) or with peripheral CD4 count $\left(\mathrm{CD} 1 \mathrm{c}^{+}\right.$mDCs: $r=-0.12, P=0.60$; CD1c ${ }^{\text {neg }}$ mDCs: $r=-0.28, P=0.25$ ). Although pDCs are known to be directly activated by HIV $-1,{ }^{22}$ no direct associations were observed between the number of $\mathrm{CD} 40^{+} \mathrm{pDCs}$ and either mucosal
( $r=0.16, P=0.50)$ or plasma viral load $(r=0.03, P=0.88)$ or with peripheral CD4 count $(r=0.09, P=0.69)$.

\section{Colonic and systemic T-cell activation correlate with colonic $\mathrm{mDC}$ activation}

Activated colon $\mathrm{CD}^{+}$and $\mathrm{CD} 8{ }^{+}$T-cell frequencies were increased in HIV-1-infected subjects (Figure 3a,b, Supplementary Table S2), and CD40 expression levels on CD1c ${ }^{+}$ $\mathrm{mDCs}$ strongly associated with the number of activated colonic $\mathrm{CD}^{+}{ }^{+}$and $\mathrm{CD}^{+}{ }^{+} \mathrm{T}$ cells. Similar but weaker associations were noted between $\mathrm{CD} 1 \mathrm{c}^{\text {neg }} \mathrm{mDC}$ activation and activated colonic $\mathrm{T}$ cells (Figure 3a,b).

A larger infiltrate of mononuclear cells was measured by histology in the colonic LP of HIV-1-infected subjects relative to control subjects, and CD40 expression levels on both $\mathrm{CD} 1 \mathrm{c}^{+}$ and $\mathrm{CD} 1 \mathrm{c}^{\text {neg }} \mathrm{mDCs}$ were positively associated with the degree of mononuclear cell infiltration (Figure 3c).

Significantly higher percentages of activated blood CD4 and CD8 T cells were found in HIV-1-infected subjects relative to uninfected controls, as expected (Figure 4a,b, Supplementary Table S2). Blood T-cell activation frequencies were positively associated with activation levels of $\mathrm{CD} 1 \mathrm{c}^{+}$but not of $\mathrm{CD} 1 \mathrm{c}^{\text {neg }}$ mDCs (Figure 4a,b).

Significant decreases in the frequencies of colonic Th1, Th17, and Th22 cells in conjunction with increased frequencies of IFN- $\gamma^{+}$CD8 T cells were observed in HIV-1-infected subjects compared with controls (Supplementary Table S3). However, 

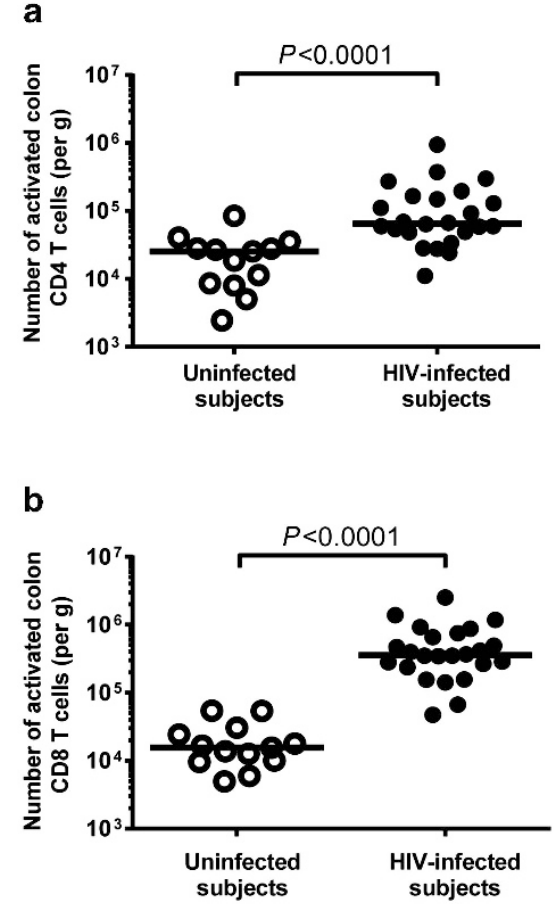

C

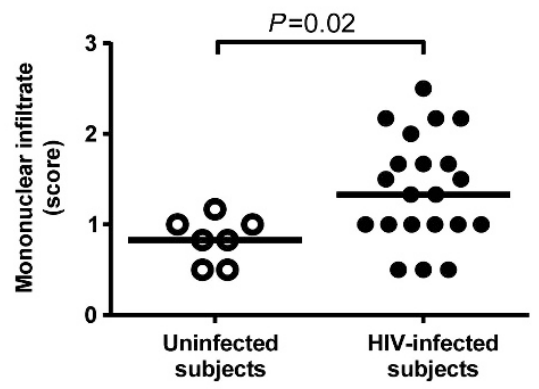

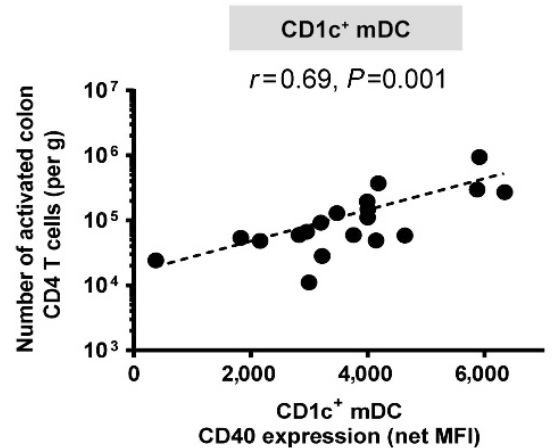
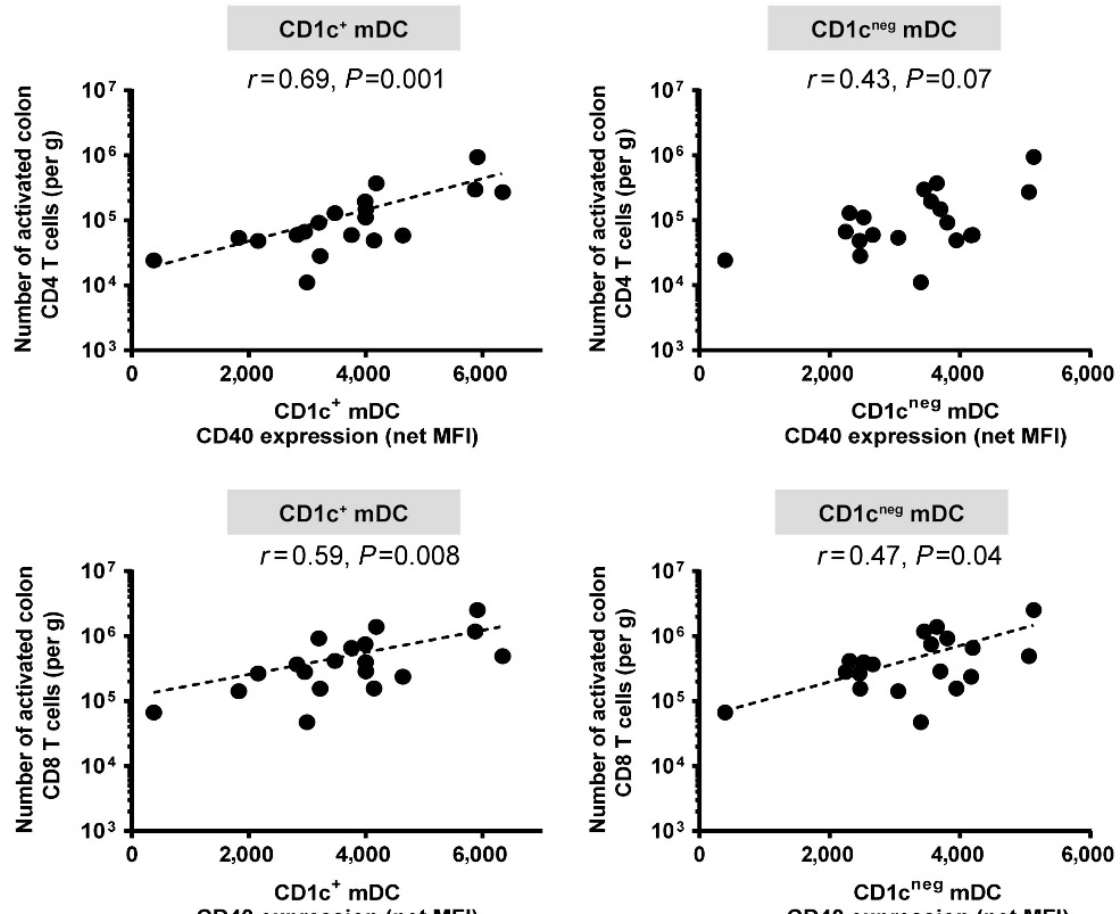

CD40 expression (net MFI)

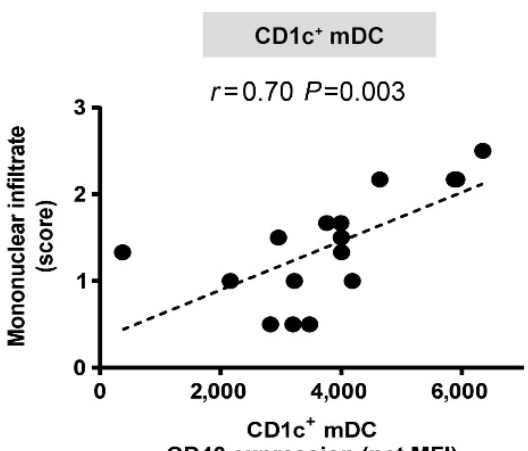

CD40 expression (net MFI)

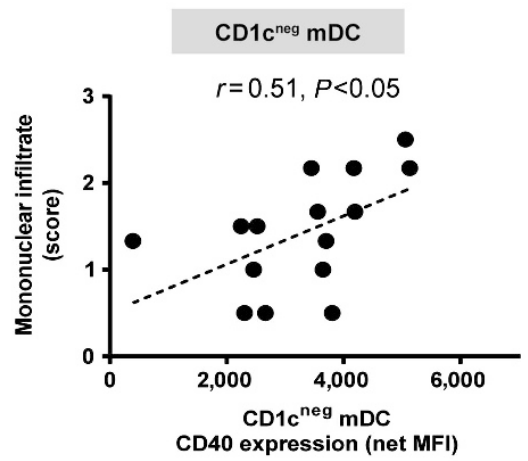

Figure 3 Activated colon $\mathrm{CD}_{1} \mathrm{c}^{+}$myeloid dendritic cells (mDCs) correlate with mucosal T-cell activation and mononuclear infiltration. Multi-color flow cytometry techniques were used to determine frequencies of activated (percentage of CD38 ${ }^{+}$HLA-DR ${ }^{+}$) colonic mucosal CD4 and CD8 T cells and hematoxylin and eosin staining to evaluate lamina propria (LP) infiltration of mononuclear cells in uninfected (open circles) and HIV-1-infected (HIVinfected; closed circles). Frequencies of colonic mucosal (a) CD38 ${ }^{+} \mathrm{HLA}-\mathrm{DR}{ }^{+}$CD4 T cells and (b) CD38 ${ }^{+} \mathrm{HLA}^{-D R}{ }^{+}$CD8 T cells (uninfected $n=13$; HIV-infected $n=24$ ) were evaluated (with background isotype values removed) as a percentage of viable, CD45 ${ }^{+}$leucocytes and converted into a total number of activated CD4 or CD8 T cells per $\mathrm{g}$ of tissue. Lines represent median values and statistical analysis was performed using Mann-Whitney test. Correlations between CD40 expression levels (mean fluorescence intensity (MFI)) on $\mathrm{CD}_{1} \mathrm{c}^{+}$and $\mathrm{CD} 1 \mathrm{c}^{\text {neg }}$ mDCs (shown with background isotype values removed; net MFI) and activated (a) CD4 T cells or (b) CD8 T cells (shown with background isotype values removed) in HIV-infected subjects $(n=19)$ were performed using Spearman's test. Dotted line is a visual representation of the significant associations. (c) Mononuclear infiltrate assessed as the relative cellularity of the LP infiltrate consisting of lymphocytes, plasma cells, eosinophils and occasional neutrophils and scored on a scale of $0=$ not present, minimal $=0.5$, mild $=1$, moderate $=2$, and severe $=3$. Values are shown as the average score of three sections of colon biopsy from uninfected (open circles, $n=7$ ) and HIV-1-infected (HIV-infected; $n=21$ ) subjects. Lines represent median values and statistical analysis was performed using Mann-Whitney test. Correlations between CD40 expression levels (MFI) on CD1c ${ }^{+}$and CD1c ${ }^{\text {neg }} \mathrm{mDCs}$ (shown with background isotype values removed; net MFI) and mononuclear infiltrate scores in HIV-infected subjects $(n=16)$ were performed using Spearman's test. Dotted line is a visual representation of the significant associations.

no significant associations were found between the levels of $\mathrm{mDC}$ activation and frequencies of cytokine-producing CD4 or CD8 T cells (data not shown).

\section{Colonic mDC activation is associated with mucosal and plasma cytokine production}

$\mathrm{CD} 40$ expression on $\mathrm{CD}^{+} \mathrm{c}^{+} \mathrm{mDC}$ strongly associated with levels of a number of inflammatory mucosal cytokines, including
IL-23, IL-1 $\beta$, IL-6, and tumor necrosis factor (TNF)- $\alpha$ as well as with IL-10 levels, in HIV-1-infected subjects (Table 2). Moreover, $\mathrm{CD}_{1 \mathrm{c}^{+}} \mathrm{mDC}$ activation positively associated with both mucosal IFN- $\gamma$ and IL-17 production. Weaker but significant associations were observed between $\mathrm{CD}_{1} \mathrm{c}^{\text {neg }} \mathrm{mDC}$ activation levels and mucosal levels of IL-23 and IFN- $\gamma$.

Plasma IL-6 levels were significantly increased in HIV-1infected subjects $\left(1.43 \mathrm{pg} \mathrm{ml}^{-1}, 0.43-5.09 ; n=24\right)$ compared 
a

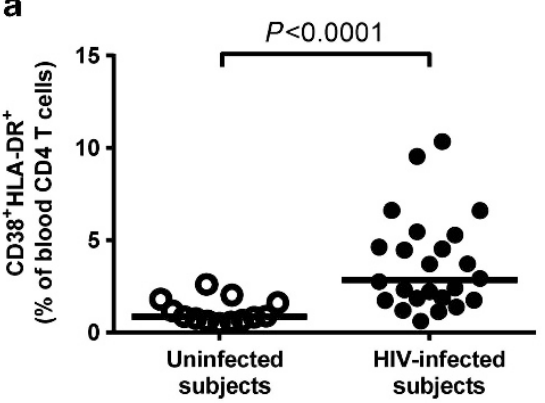

b

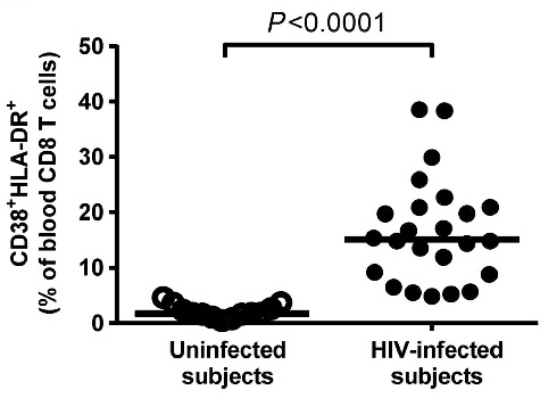

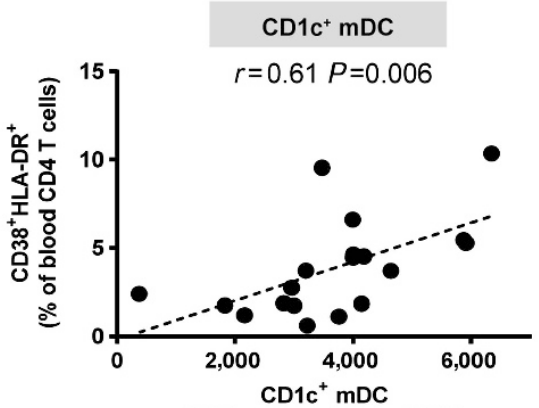

CD40 expression (net MFI)

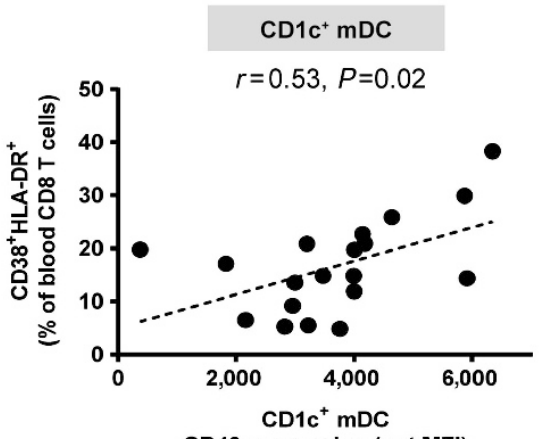

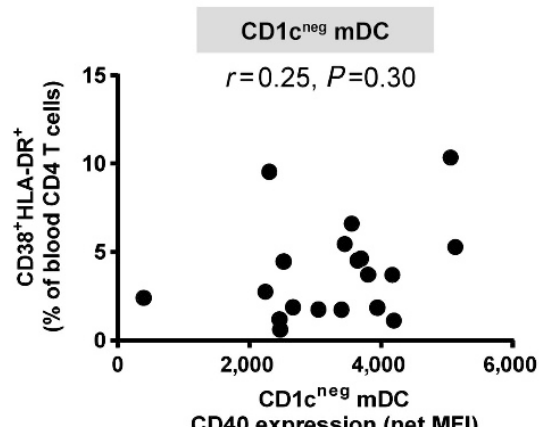

CD40 expression (net MFI)

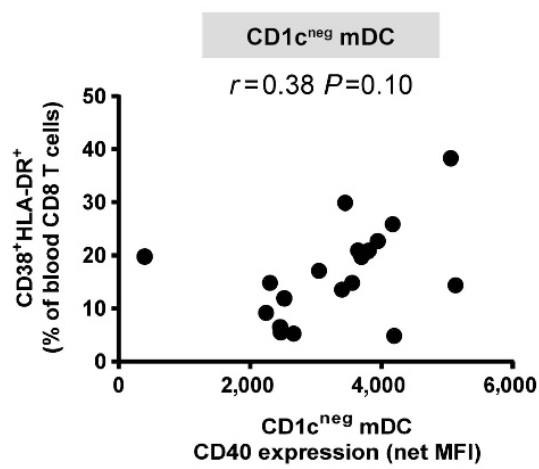

Figure 4 Activated colon $\mathrm{CD}_{\mathrm{c}} \mathrm{c}^{+}$myeloid dendritic cells (mDCs) correlate with systemic T-cell activation. Multi-color flow cytometry techniques were used to determine frequencies of activated (percentage of CD38 ${ }^{+} \mathrm{HLA}^{-D R}{ }^{+}$) blood CD4 and CD8 T cells in uninfected (open circles; $n=13$ ) and HIV-1infected (HIV-infected; $n=24$; closed circles). Percentages of (a) CD38 ${ }^{+} \mathrm{HLA}^{+} \mathrm{DR}{ }^{+}$blood CD4 T cells and (b) $\mathrm{CD}_{3}{ }^{+} \mathrm{HLA}^{-D R}{ }^{+}$blood CD8 T cells as a fraction of blood CD4 or CD8 T cells (shown with background isotype values removed). Lines represent median values and statistical analysis was performed using Mann-Whitney test. Correlations between CD40 expression levels (mean fluorescence intensity (MFI)) on CD1c ${ }^{+}$and CD1c ${ }^{\text {neg }} \mathrm{mDCs}$ (shown with background isotype values removed; net MFI) and activated blood (a) CD4 and (b) CD8 T cells (shown with background isotype values removed) in HIV-infected subjects $(n=19)$ were performed using Spearman's test. Dotted line is a visual representation of the significant associations.

Table 2 Activated CD1c ${ }^{+}$myeloid dendritic cell $(\mathrm{mDC})$ or CD1c neg $\mathrm{mDC}$ associations with constitutive mucosal cytokines in HIV1-infected subjects

\begin{tabular}{|c|c|c|c|}
\hline & 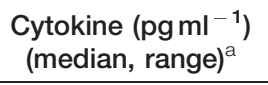 & $\begin{array}{l}\text { CD40 expression levels } \\
\text { (MFI) on } \mathrm{CD}^{+} \mathrm{c}^{+} \mathrm{mDC}(n=15)\end{array}$ & $\begin{array}{c}\text { CD40 expression levels } \\
\text { (MFI) on CD1c }{ }^{\text {neg }} \mathrm{mDC}(n=15)\end{array}$ \\
\hline IL-23 & $47.7,0-1264$ & $r=0.67, P=0.008$ & $r=0.55, P=0.03$ \\
\hline IL-6 & $513.1,0-8711$ & $r=0.68, P=0.006$ & $r=0.38, P=0.16$ \\
\hline TNF- $\alpha$ & $48.9,0-2301$ & $r=0.66, P=0.009$ & $r=0.40, P=0.14$ \\
\hline$\underline{\mathrm{IL}-17}$ & $11.6,0-125.5$ & $r=0.56, P=0.03$ & $r=0.27, P=0.33$ \\
\hline
\end{tabular}

IFN, interferon; IL, interleukin; MFI, mean fluorescence intensity; TNF, tumor necrosis factor. Statistical analysis was performed using Spearman's test. Bold values highlight statistically significant correlations.

${ }^{\mathrm{a}} \mathrm{N}=19 \mathrm{HIV}$-1-infected subjects.

with controls $\left(0.70 \mathrm{pg} \mathrm{ml}^{-1}, 0.19-2.16 ; n=14 ; P=0.002\right)$; however, no significant correlation between $\mathrm{mDC}$ activation and IL-6 levels was observed in HIV-1-infected subjects $(r=0.08, P=0.76)$. Plasma levels of other cytokines were evaluated in a subset of HIV-1-infected subjects $(n=18)$. TNF$\alpha$ levels strongly associated with plasma HIV-1 viral load $(r=0.62, P=0.006)$ and with plasma IL-6 $(r=0.64, P=0.004)$ and sCD14 levels $(r=0.79, P=0.001)$. IL-10 levels also associated with plasma HIV-1 viral load $(r=0.69, P=0.001)$ and with $\mathrm{sCD} 14$ ( $r=0.73, P=0.0007)$. TNF- $\alpha$ and IL-10 levels strongly correlated with each other $(r=0.87, P<0.0001)$. $\mathrm{CD}_{1} \mathrm{c}^{+} \mathrm{mDC}$ activation was positively associated with plasma levels of TNF- $\alpha(r=0.63, P=0.02 ; n=14)$ and IL-10 $(r=0.76$, $P=0.002 ; n=14)$, whereas $\mathrm{CD} 1 \mathrm{c}^{\text {neg }} \mathrm{mDC}$ activation was associated with plasma IFN- $\gamma(r=0.59, P=0.03 ; n=14)$ and weakly with IL-10 $(r=0.54, P=0.05 ; n=14)$. 

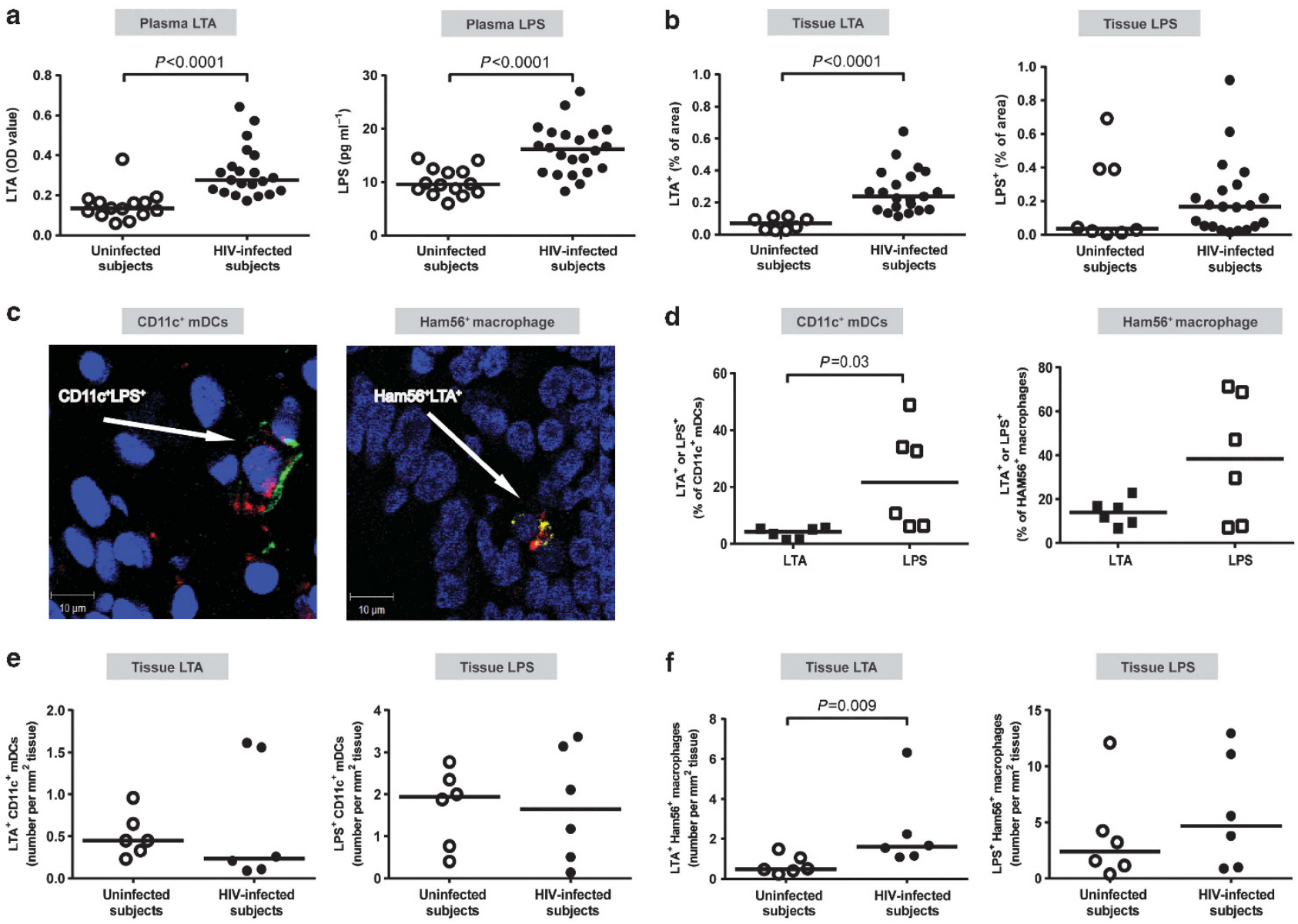

Figure 5 Colonic tissue and systemic levels of microbial products are increased in HIV-1-infected subjects and colonic myeloid dendritic cells (mDCs) associate with tissue lipopolysaccharides (LPS) to a greater extent than lipoteichoic acid (LTA). Levels of LTA and LPS were evaluated in the (a) plasma of uninfected $(n=14)$ and HIV-1-infected (HIV-infected; LTA $n=21$; LPS $n=22)$ subjects and in the (b) colonic lamina propria (LP) of uninfected $(n=8)$ and HIV-1-infected (HIV-infected, $n=21$ ) subjects. Lines represent median values and statistical analysis was performed using Mann-Whitney test. (c) Representative images demonstrating localization of mDCs (CD11c/green) or macrophages (Ham56/yellow) with either LTA or LPS (red) in formalin-fixed, paraffin-embedded colon biopsy tissue of an HIV-1-infected subject. (d) Comparisons between the percentages of LTA ${ }^{+}$or LPS $^{+}$ $\mathrm{CD}_{11 \mathrm{c}^{+}} \mathrm{mDCs}$ and Ham56 ${ }^{+}$macrophages in HIV-infected subjects $(n=6)$. Lines represent median values and statistical analysis was performed using Wilcoxon's matched-pairs signed rank test. (e, f) The number of $\mathrm{LTA}^{+}$or LPS ${ }^{+}(\mathbf{e}) \mathrm{CD}_{11 \mathrm{c}^{+}} \mathrm{mDC}$ and $(\mathbf{f}) \mathrm{Ham} 6^{+}$macrophages per mm ${ }^{2}$ of tissue in uninfected $(n=6)$ and HIV-infected subjects $(n=6)$. Lines represent median values and statistical analysis was performed using Mann-Whitney test.

\section{Colonic mDCs are identified in association with tissue LPS to a greater extent than lipoteichoic acid (LTA)}

Levels of Gram-negative bacterial LPS in the plasma of HIV-1infected subjects were increased relative to controls (Figure 5a). In agreement with the early studies, ${ }^{6}$ plasma LPS levels in HIV1 -infected subjects correlated with blood CD4 $(r=0.62$, $P=0.002)$ and $\operatorname{CD} 8(r=0.41, P=0.058)$ T-cell activation. Moreover, plasma LPS levels significantly associated with the levels of mucosal IL-1 $\beta(r=0.58, P=0.02)$ and IL-6 $(r=0.54$, $P=0.03)$ and weakly with mucosal TNF- $\alpha \quad(r=0.47$, $P=0.058)$. Increased plasma levels of the Gram-positive cell wall component LTA were also observed in HIV-1-infected subjects compared with controls (Figure 5a). However, LTA levels correlated only with blood CD4 T-cell activation $(r=0.47, \quad P=0.03)$. No significant associations between these indicators of systemic MT and activated colonic $\mathrm{CD}^{+} \mathrm{c}^{+} \mathrm{mDCs}$ (LTA $(n=16): r=-0.16, P=0.55$; LPS $(n=17): \quad r=0.22, \quad P=0.39)$ or $\mathrm{CD} 1 c^{\text {neg }}$ mDCs (LTA: $r=-0.31, \quad P=0.23$; LPS: $\quad r=-0.03, \quad P=0.91)$ were observed in HIV-1-infected subjects.

Tissue LTA and LPS levels were both higher in HIV-1infected subjects compared with control subjects, but only LTA levels reached statistical significance (Figure 5b). In HIV-1infected subjects, no significant associations were found between tissue LTA or LPS levels and CD1c ${ }^{+} \mathrm{mDC}$ (LTA $(n=14): \quad r=-0.14, \quad P=0.63 ; \quad$ LPS $(n=14): r=-0.09$, $P=0.76$ ) or $\mathrm{CD} 1 c^{\text {neg }} \mathrm{mDC}$ (LTA: $r=0.21, P=0.47$; LPS: $r=15, P=0.61)$ activation.

In HIV-1-infected subjects, a greater fraction of CD11c ${ }^{+}$ mDCs and HAM56 ${ }^{+}$macrophages were associated with LPS than with LTA, although this did not reach statistical significance for macrophages (Figure $\mathbf{5 c}, \mathbf{d}$ ). Similar trends were observed in uninfected subjects (Figure 5e,f). Both LTA and LPS were more frequently associated with macrophages 


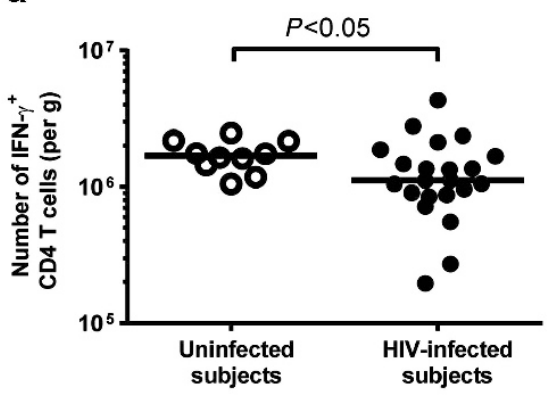

b

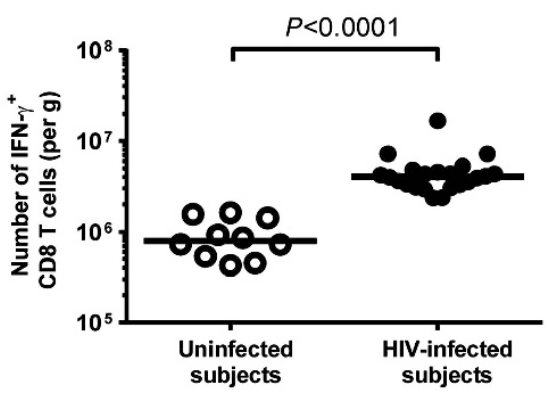

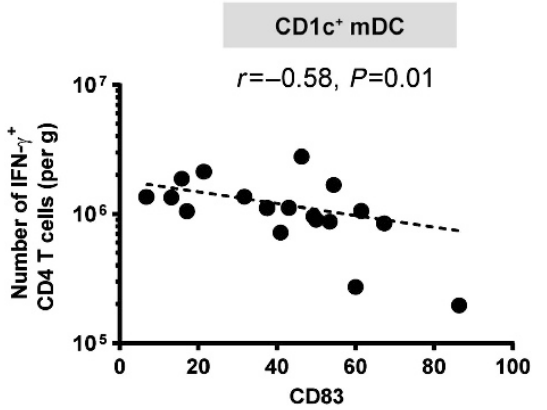

$\left(\%\right.$ of $\left.\mathrm{CD}^{+} \mathrm{c}^{+} \mathrm{mDC}\right)$

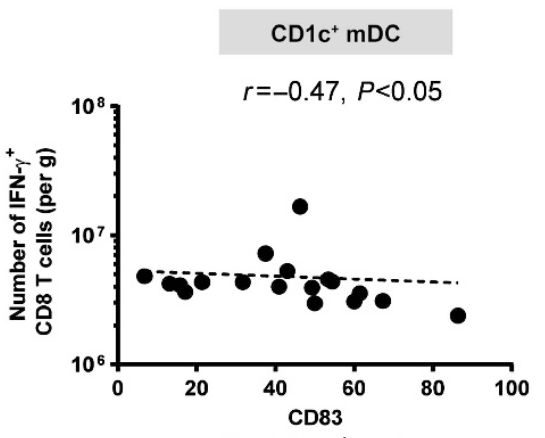

$\left(\%\right.$ of $\left.\mathrm{CD}^{+} \mathrm{c}^{+} \mathrm{mDC}\right)$

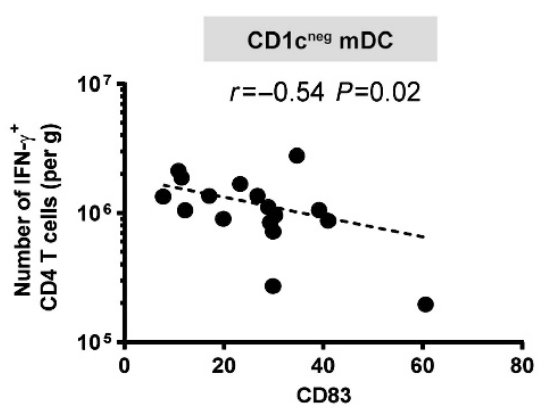

$\left(\%\right.$ of $\left.\mathrm{CD} 1 \mathrm{c}^{\mathrm{neg}} \mathrm{mDC}\right)$

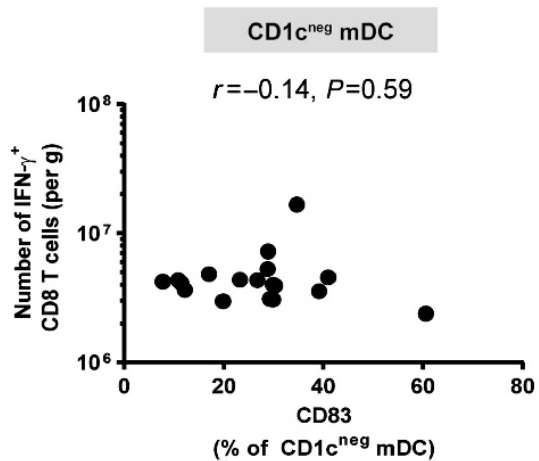

Figure $6 \mathrm{CD}^{2} 3^{+}$myeloid dendritic cells (mDCs) negatively correlate with colonic IFN- $\gamma$-producing CD4 and CD8 T cells. Multi-color flow cytometry techniques were used to determine the frequencies of IFN- $\gamma$-producing colonic CD4 and CD8 T cells following mitogenic stimulation in uninfected (open circles; $n=10$ ) and HIV-1-infected (HIV-infected; $n=22$ ) subjects. Frequencies of colonic (a) IFN- $\gamma^{+}$CD4 T cells and (b) IFN- $\gamma^{+}$CD8 T cells were evaluated (background isotype values removed) as a percentage of viable, CD45 ${ }^{+}$leucocytes and converted into a total number of activated CD4 or CD8 $T$ cells per $\mathrm{g}$ of tissue. Lines represent median values and statistical analysis was performed using Mann-Whitney test. Correlations between percentage of $\mathrm{CD}_{3} 3^{+} \mathrm{CD} \mathrm{c}^{+}$and CD1c neg mDCs (shown with background isotype values removed) and the number of IFN- $\gamma^{+}$(a) CD4 T cells or (b) CD8 T cells (shown with background isotype values removed) in HIV-infected subjects $(n=18)$ were performed using Spearman's test. Dotted line is a visual representation of the significant associations.

than with mDCs. When the numbers of $\mathrm{LTA}^{+}$or $\mathrm{LPS}^{+}$mDCs were compared between HIV-1-infected and uninfected subjects, no significant differences were observed $(P=0.46$ and $P=0.90$, respectively) (Figure 5e). More $\mathrm{LTA}^{+}$macrophages were observed in the LP of HIV-1-infected subjects than in uninfected subjects, whereas the number of LPS ${ }^{+}$macrophages was similar between the two cohorts (Figure 5f).

Percentage of CD83-expressing colonic mDCs is negatively associated with IFN- $\gamma$-producing colonic $\mathrm{T}$ cells

In HIV-1-infected subjects, the percentage of $\mathrm{CD} 83^{+} \mathrm{CD} 1 \mathrm{c}^{+}$ mDCs negatively correlated with the number of IFN- $\gamma$ producing colonic CD4 and CD8 T cells, whereas the percentage of $\mathrm{CD} 83^{+} \mathrm{CD} 1 \mathrm{c}^{\text {neg }} \mathrm{mDC}$ negatively associated only with IFN- $\gamma^{+}$CD4 T cells (Figure 6a,b). A significant correlation between the percentage of $\mathrm{CD} 83^{+}$pDCs and the percentage of IFN- $\gamma$-producing $\mathrm{CD}^{+} \mathrm{T}$ cells $(r=0.53$, $P=0.02 ; n=20)$ was noted; however, no association with the absolute number of IFN- $\gamma^{+} \mathrm{CD} 8{ }^{+} \mathrm{T}$ cells or with any other immunological or virological parameters were observed.

\section{Abundances of altered mucosal bacterial species are associated with colonic mDC activation}

We previously evaluated mucosal and fecal microbiomes to the genus level in a subset of study subjects ${ }^{16}$ and have now identified 21 mucosa-associated bacterial species, based on $99 \%$ identity to sequences in the SILVA database ${ }^{23}$ that are significantly over (6) or under (15) represented in HIV-1-infected subjects, termed "HIV-altered mucosal bacteria" (HAMB) species (Supplementary Table S4). Similar to results in our previous study, ${ }^{16}$ greater abundance of the two Proteobacteria spp. was noted only in the mucosa, whereas Prevotella species abundance was significantly greater in both mucosa and stool of HIV-1-infected subjects compared with controls.

Levels of CD40 on CD1c ${ }^{+}$mDCs trended $(P<0.1)$ toward positive associations with high-abundance Prevotella copri and $P$. stercorea and toward negative associations with lowabundance Bacteroides acidifaciens, Blautia schinikii, and Rumminococcus bromii (Table 3). CD1c neg $\mathrm{mDC}$ activation also trended toward a negative association with $R$. bromii, but no clear associations with any other HAMB species were noted (Table 3).

High-abundance HAMB species induce greater cytokine ${ }^{+}$ $\mathrm{CD}^{+} \mathrm{C}^{+} \mathrm{mDC}$ frequencies in vitro compared with lowabundance HAMB species

Production of IL-23, IL-1 $\beta$, and IL-10 by $\mathrm{CD}_{1}{ }^{+} \mathrm{mDC}$ following in vitro stimulation of total LP mononuclear cells (LPMC) with $P$. copri and $P$. stercorea (high-abundance HAMB) and R. bromii (low-abundance HAMB) was assessed. These cytokines and HAMB were specifically chosen based on their in vivo associations with $\mathrm{CD}^{+} \mathrm{c}^{+}$activation. 
Table 3 Activated CD1c ${ }^{+}$myeloid dendritic cell (mDC) or CD1c ${ }^{\text {neg }} \mathrm{mDC}$ associations with HIV-altered mucosal bacteria (HAMB) species

\begin{tabular}{|c|c|c|c|c|}
\hline HAMB species & Prevalence $^{a}$ & $\begin{array}{c}\uparrow \text { or } \downarrow \text { in HIV-1-infected } \\
\text { subjects }^{b}\end{array}$ & $\begin{array}{l}\text { CD40 expression levels } \\
\text { (MFI) on } \mathrm{CD}^{\circ} \mathrm{c}^{+} \mathrm{mDC}^{\mathrm{C}}\end{array}$ & 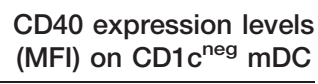 \\
\hline Prevotella copri & $100 \%$ & $\uparrow(P=0.02)$ & $\boldsymbol{r}=0.55, P=0.0525$ & $r=0.16, P=0.60$ \\
\hline Bacteroides acidifaciens & $58.8 \%$ & $\downarrow(P=0.02)$ & $r=-0.51, P=0.0592$ & $r=-0.29, P=0.28$ \\
\hline Blautia schinikii & $52.9 \%$ & $\downarrow(P=0.005)$ & $r=-0.45, P=0.0863$ & $r=-0.21, P=0.38$ \\
\hline
\end{tabular}

$\mathrm{MFI}$, mean fluorescence intensity.

Bold values highlight trending correlations $(P<0.1)$.

aPercentage of HIV-1-infected subjects with each species. Given the small samples size, statistical analysis to determine associations with activated colonic mDC was only assessed when each species was detected in $>50 \%$ of HIV-1-infected subjects.

${ }^{b}$ Increased $(\uparrow)$ or decreased $(\downarrow)$ abundance of each bacteria in HIV-1-infected subjects $(n=17)$ compared with uninfected controls $(n=14)$ determined using Mann-Whitney test.

${ }^{\mathrm{c}}$ Associations between activated colonic $\mathrm{CD} \mathrm{c}^{+} \mathrm{mDC}(n=13)$ or $\mathrm{CD} 1 \mathrm{c}^{\text {neg }} \mathrm{mDC}(n=13)$ and abundance of HAMB were determined using Spearman's test.
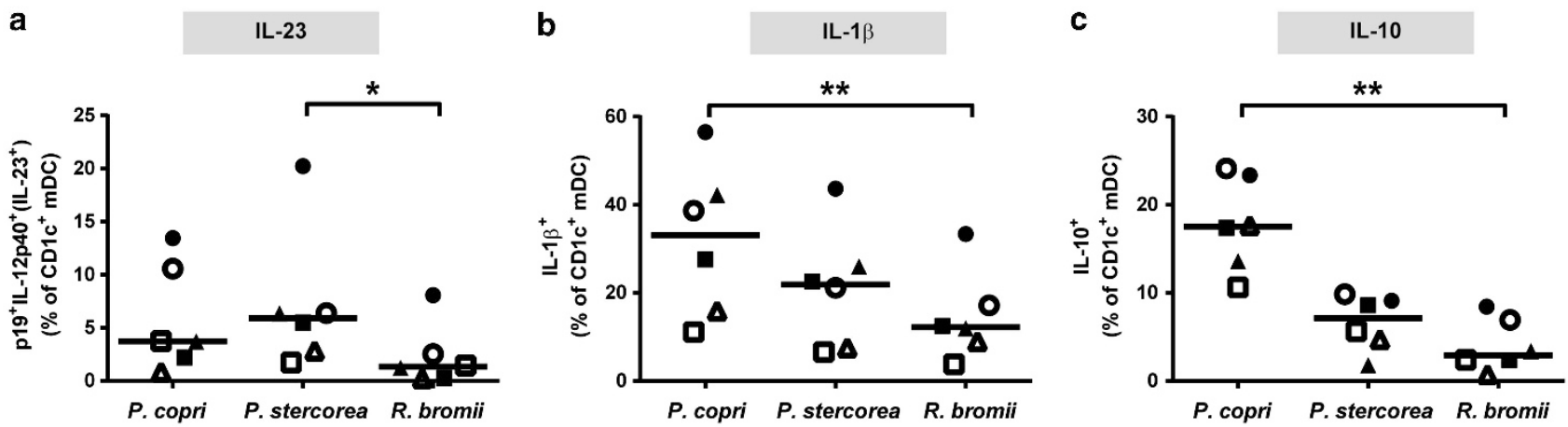

Figure $7 \mathrm{CD} \mathrm{c}^{+}$myeloid dendritic cell $(\mathrm{mDC})$ cytokine production in response to in vitro stimulation with HIV-altered mucosal bacteria (HAMB) species Colonic lamina propria mononuclear cells ( $n=7$ samples) were exposed to Prevotella copri, P. stercorea or Ruminococcus bromii for $18-20 \mathrm{~h}$, and multicolor intracellular cytokine flow cytometry techniques were used to enumerate (a) IL-23 ${ }^{+}\left(\mathrm{IL}-12 \mathrm{p} 40^{+} \mathrm{p} 19^{+}\right),(\mathbf{b}) \mathrm{IL}-1 \beta^{+}$, and (c) IL-10 ${ }^{+} \mathrm{CD} 1 \mathrm{c}^{+} \mathrm{mDCs}$. Appropriate isotype controls were removed to control for background staining. Values are shown as HAMB-specific cytokine ${ }^{+} \mathrm{mDCs}$ determined by removing the percentage of cytokine ${ }^{+} \mathrm{CD}_{1} \mathrm{c}^{+} \mathrm{mDC}$ s detected in unstimulated cultures. Each symbol is a unique donor. Lines represent median values and statistical analysis was performed using Friedman's test for matched-paired comparisons across multiple groups, with Dunn's multiple comparison test performed when the overall $P$-value was $<0.05$. ${ }^{\star} P<0.05,{ }^{\star \star} P<0.01$.

Exposure to each of the HAMB induced significant frequencies of IL-23-, IL-1 $\beta$-, and IL-10-producing CD1c ${ }^{+}$ mDCs, indicating that all three HAMB species activate colonic $\mathrm{CD}_{1}{ }^{+} \mathrm{mDCs}$ in vitro to some degree (Supplementary Table S5). P. copri and P. stercorea induced a higher percentage of IL$23^{+} \mathrm{CD} \mathrm{c}^{+}$mDCs compared with $R$. bromii, with this reaching statistical significance for $P$. stercorea (Figure 7a). $P$. copri induced the highest fraction of $\mathrm{IL}_{-} 1 \beta^{+} \mathrm{CD} 1 \mathrm{c}^{+} \mathrm{mDCs}$, and this difference was highly significant $(P<0.01)$ when compared with $R$. bromii (Figure $7 \mathbf{b}$ ).

P. copri induced the highest percentage of $\mathrm{IL}-10^{+} \mathrm{CD} 1 \mathrm{c}^{+}$ mDCs, which was, on average, $3.3 \times$ and $7.7 \times$ that induced by $P$. stercorea and R. bromii, respectively, and reached statistical significance $(P<0.01)$ relative to $R$. bromii (Figure $7 \mathrm{c}$ ). In response to $P$. copri stimulation, a small fraction of IL- $10^{+} \mathrm{CD} \mathrm{c}^{+}$mDC co-produced IL-23 (mean $8.2 \pm 3.9 \%$ (s.e.m.)), whereas a higher percentage of $\mathrm{IL}^{-10^{+}} \mathrm{CD} 1 \mathrm{c}^{+}$ $\mathrm{mDC}$ co-produced $\mathrm{IL}-1 \beta^{+}(62 \pm 6.1 \%)$, suggesting that
IL-23-producing $\mathrm{CD} 1 \mathrm{c}^{+} \mathrm{mDC}$ are a separate population of cells to those producing IL-10.

\section{DISCUSSION}

To our knowledge, this is the first study to address whether colonic $\mathrm{mDC}$ phenotype and function is altered in chronic untreated HIV-1 infection. In agreement with a number of previous studies in pathogenic simian immunodeficiency virus (SIV) infection, ${ }^{24-27}$ no significant differences were observed in the frequency of colon $\mathrm{CD} 1 \mathrm{c}^{+}$or CD1c $\mathrm{c}^{\text {neg }} \mathrm{mDCs}$ in HIV-1infected subjects compared with uninfected controls. However, HIV-1 infection induced an activated but dysregulated intestinal $\mathrm{mDC}$ phenotype characterized by increased levels of CD40 expression and decreased CD83 expression, similar to what we previously described for lymph node DCs during HIV infection. $^{21}$

We show for the first time that specific Prevotella species, increased in the stool and colonic mucosa of HIV-1-infected 
subjects, correlated in abundance with colonic $\mathrm{mDC}$ activation levels in vivo and also had the capability of inducing strong proinflammatory cytokine production by colonic mDCs in vitro. These findings expand upon our previous observations that, in HIV-1-infected subjects, mucosal abundance of Prevotella spp. (genus level) was associated both with $\mathrm{CD} 1 \mathrm{c}^{+} \mathrm{mDC}$ activation and with colon CD4 and CD8 T-cell activation. ${ }^{16}$ Taken together, these results suggest that increased abundance of these pathobiont bacterial species in the intestinal mucosa may contribute to HIV-associated mucosal inflammation and immune activation, supporting previous studies that demonstrated the "pathogenic potential" of Prevotella species in periodontal disease, ${ }^{28}$ ulcerative colitis, ${ }^{29}$ and arthritis. ${ }^{30}$

Although typically indicative of "maturation", the precise role for DC expression of CD83 in directing immune responses is not well understood. Downregulation of membrane-bound CD83 by RNA interference ${ }^{31,32}$ or by viruses such as human cytomegalovirus $^{33}$ and herpes simplex virus type $1^{34}$ on human blood DCs resulted in decreased T-cell stimulatory capacity. However, fewer $\mathrm{CD} 83^{+}$cells were detected in the inflamed areas of colonic and ileal Crohn's disease samples compared with control and uninflamed areas, ${ }^{35}$ suggesting that in the intestinal mucosa CD83 may have regulatory effects. This concept of CD83-mediated mucosal regulation is further supported by our observation that, in HIV-1-infected subjects, frequencies of colonic $\mathrm{CD} 83^{+} \mathrm{mDCs}$ were inversely associated with IFN- $\gamma$-producing colonic T cells. However, further studies are warranted to determine the mechanistic relationship between CD83-expressing mucosal $\mathrm{mDC}$ and IFN- $\gamma$-producing $\mathrm{T}$ cells and to evaluate whether this is an $\mathrm{mDC}$-mediated process or, conversely, IFN- $\gamma$-producing $\mathrm{T}$ cells have a role in modulating intestinal $\mathrm{mDC}$ activation during HIV-1 infection.

A potential "central role" for activated colonic $\mathrm{mDC}$ in HIVassociated pathogenesis is further highlighted by our observations that CD40 expression levels on $\mathrm{CD} 1 \mathrm{c}^{+}$mDCs positively correlated with colonic CD4 and CD8 T-cell activation. Further, $\mathrm{CD} 1 \mathrm{c}^{+} \mathrm{mDC}$ activation also associated with blood CD4 and CD8 T cells activation, thereby linking colon mDC activation to a marker of HIV-1 disease progression. ${ }^{2,3}$ Moreover, activated CD1c ${ }^{+}$mDCs in HIV-1-infected subjects was associated with numerous mucosal cytokines, including IL23 and IL-1 $\beta$. Within the mucosa, increased levels of IL-23 and IL-1 $\beta$ have been implicated in intestinal inflammation mediated, in part, through the promotion of T-cell-associated IFN- $\gamma$ and IL-17 production. ${ }^{36,37}$ In our study, levels of CD40 expression on colonic $\mathrm{mDC}$ s were also associated with mucosal levels of IFN- $\gamma$ and IL-17, suggesting an intricate relationship between $\mathrm{mDC}$ activation, mucosal T-cell activation, and cytokine production in the setting of HIV-1 infection. These in vivo observations expand on our previous in vitro study that demonstrated a requirement for LP $\mathrm{mDCs}$ in the in vitro expansion and enhanced infection of Th1 and Th17 cells in response to exposure to commensal bacteria and HIV-1. ${ }^{19}$ Although we did not see direct correlations between $\mathrm{mDC}$ activation levels and absolute Th1 or Th17 frequencies, this finding may be due to the fact that these mucosal Th subsets are depleted early in the course of HIV infection ${ }^{38}$ and thus absolute Th cell numbers might not be expected to reflect ongoing mucosal inflammation during chronic disease.

Intriguingly, HIV-1-associated colonic mDC activation levels positively associated with mucosal and systemic IL-10 production, a cytokine with well described immuno-regulatory functions. ${ }^{39}$ Increased levels of IL-10, in conjunction with increased levels of pro-inflammatory cytokines, have been reported in both acute and chronic HIV-1 infection. ${ }^{40,41}$ Systemically administered IL-10 stimulated the production of IFN- $\gamma$ during human endotoxemia, ${ }^{42}$ suggesting that IL-10 can have pro-inflammatory effects, especially concurrent with exposure to microbial products. IL-10 regulates production of IL-23 by human blood mDCs in response to commensal bacteria in vitro ${ }^{43}$ and a similar negative feedback mechanism to compensate for increased production of pro-inflammatory IL-23 by intestinal $\mathrm{mDCs}$ in response to translocating commensal bacteria may be at play in the colon of HIV-1infected subjects. Indeed, we observed production of IL-10 by $\mathrm{CD}_{1 \mathrm{c}^{+}} \mathrm{mDC}$ in response to in vitro exposure to HAMB, and these DCs were a different population to those producing IL-23. Although our current in vivo and in vitro observations suggest a role for intestinal mDCs in IL-10 production, the exact nature of the immune-regulatory vs. pro-inflammatory effects of IL-10 in the setting of HIV-1 infection requires further investigation.

Estes et al. ${ }^{44}$ utilized quantitative image analysis to directly demonstrate translocation of LPS and Escherichia coli in the colon of chronically SIV-infected rhesus macaques, and increased levels of MT were due, in part, to ineffective phagocytosis by intestinal macrophages. To our knowledge, no studies have quantitated levels of microbial products in the human colonic LP during chronic untreated HIV-1 infection nor evaluated the co-localization of microbial products with resident LP mDCs and macrophages. In our study, HIV-1infected subjects had heightened tissue levels of both LTA and LPS, although neither appeared to directly correlate with mucosal $\mathrm{mDC}$ activation. These results suggest that both Gram-negative and Gram-positive bacteria and their products are translocating even though only Gram-negative bacteria were increased in abundance in the mucosa. A greater fraction of LTA and LPS was associated with macrophages than with $\mathrm{mDCs}$, in keeping with the reported robust phagocytic ability of tissue macrophages. ${ }^{45}$ Despite an increase in LTA and LPS tissue levels and in the number of LP macrophages in HIV-1infected subjects, we only observed an increase in the number of $\mathrm{LTA}^{+}$LP macrophages in this cohort, suggesting a defect in macrophage function in the context of bacterial uptake of LPS or Gram-negative bacteria in chronic HIV-1 infection. Indeed, the ability of LP macrophages and mDCs to limit MT in the LP of both LTA and LPS must still be somewhat ineffective given the increased levels of these bacterial products in the plasma of HIV-1-infected subjects.

In a previous study, stimulation of LPMC with a synthetic TLR7/8 ligand that mimics HIV-1 ssRNA, in conjunction with a Gram-negative bacterial TLR4 ligand (LPS), resulted in a synergistic increase in IL-23 production by intestinal $\mathrm{mDC}$ 


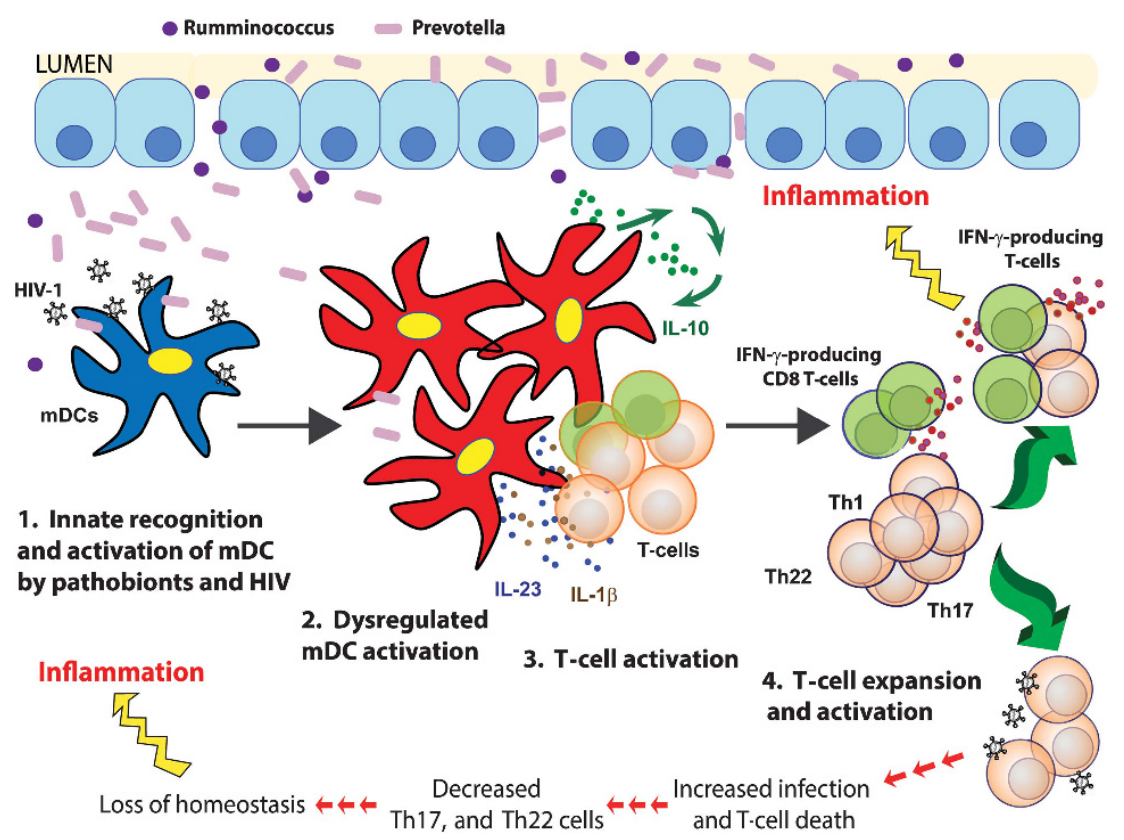

Figure 8 Proposed model illustrating colonic myeloid dendritic cells (mDCs) driving mucosal immune activation and inflammation during chronic untreated HIV-1 infection. HIV replication in the lamina propria (LP) results in epithelial barrier disruption, leading to the (1) increased translocation of Gram-negative Prevotella into the LP, which synergizes with HIV-1 to induce (2) a dysregulated mDC activation profile characterized by increased levels of CD40 and decreased CD83 expression. Activated mDCs subsequently induce (3) increased T-cell activation via stimulation of bacteria-specific CD4 T cells ${ }^{19,49}$ through cell-cell contact (e.g., CD40/CD40L), production of inflammatory cytokines (interleukin (IL)-23, IL-1ß), and potentially via CD83mediated loss of T-cell regulation. ${ }^{35} \mathrm{mDC}$ produce IL-10 to compensate for increased pro-inflammatory cytokine production; ${ }^{43}$ however, this may also exacerbate interferon (IFN)- $\gamma$ production. ${ }^{42}$ In total, this culminates in (4) increased T-cell activation and expansion of Thelper type 1 (Th1), Th17, Th22, and IFN- $\gamma$-producing CD8 T cells. Activated Th1/17/22 cells are the targets for viral replication, ${ }^{19}$ which ultimately results in their infection and depletion. Increased mucosal mDC and T-cell activation and inflammation, a loss of mDC-mediated regulation, and a lack of "protective" Th17 and Th22 cells further contribute to epithelial barrier breakdown and microbial translocation, thereby potentiating a vicious cycle that ultimately leads to systemic inflammation and immune activation and their attendant comorbidities.

in vitro. ${ }^{18}$ In this clinical study, levels of $\mathrm{CD}_{\mathrm{c}} \mathrm{c}^{+} \mathrm{mDC}$ activation correlated with mucosal HIV-1 viral load, suggesting that HIV-1 itself may have a role in intestinal $\mathrm{mDC}$ activation. Moreover, in both uninfected and HIV-1-infected subjects, the fraction of mDCs found in association with LPS was higher than that with LTA, suggesting an increased likelihood of Gram-negative bacteria directly activating colonic mDC. This finding is in keeping with our observations that Gram-negative Prevotella species abundance correlated with colonic mDC activation in vivo and that Prevotella species induced higher frequencies of cytokine-expressing $\mathrm{mDCs}$ in vitro than did Gram-positive $R$. bromii. These observations raise the possibility that HIV-1 and translocating Gram-negative bacteria act in concert to induce intestinal $\mathrm{mDC}$ activation in vivo and thereby potentiate mucosal inflammation. Further studies will be needed to understand whether HIV-1-associated activation is mediated by direct $\mathrm{HIV}-1 / \mathrm{mDC}$ interactions or in a bystander manner via effects of HIV-1 on colonic pDCs ${ }^{46}$ or other cells, as well as to identify the exact viral and bacterial determinants responsible for intestinal DC activation and cytokine production.

As part of this study, we also investigated the impact of HIV1 infection on colonic pDC frequency and activation state. A trend toward increased frequencies of intestinal pDCs, a significant increase in the numbers of $\mathrm{CD}_{4} 0^{+} \mathrm{pDCs}$, and a decrease in the percentages of CD83-expressing pDCs were observed in HIV-1-infected subjects compared with uninfected controls. Further, the numbers of activated pDCs were directly associated with the levels of $\mathrm{CD}^{+} \mathrm{c}^{+} \mathrm{mDC}$ activation, suggesting that common factors might be driving activation in both DC subsets. These observations are in keeping with recent studies demonstrating increased pDC frequencies in the ileum of chronically HIV-infected subjects ${ }^{47}$ as well as increased pDC frequencies with poly-functional cytokine phenotypes in pathogenic SIV infection models. ${ }^{25,26,48}$ Unlike mDCs, pDCs are rarely found in intestinal tissue under steady-state conditions, ${ }^{18,22}$ thus accumulation of activated pDCs during chronic HIV-1 infection is likely due to increased migration from the blood to the colon. . $^{26,26,47}$

In conclusion, we propose a model whereby colonic mDCs drive mucosal immune activation and inflammation during chronic untreated HIV-1 infection (Figure 8). Increased translocation of Gram-negative Prevotella into the LP synergizes with HIV to induce intestinal $\mathrm{mDC}$ activation. Activated $\mathrm{mDC}$ subsequently stimulate bacteria-specific CD4 T cells ${ }^{19,49}$ through cell-cell contact and production of inflammatory cytokines (IL-23, IL-1 $\beta$ ), with additional nonspecific T-cell activation potentially occurring via CD83mediated loss of mucosal $\mathrm{T}$-cell regulation. This process leads to expansion of Th1, Th17, and Th22, as well as inflammatory 
IFN- $\gamma$-producing CD8 T cells. Activated Th1/17/22 cells are targets for viral replication, ${ }^{19}$ which ultimately results in their infection and depletion. ${ }^{49}$ Increased mucosal inflammation, a loss of DC-mediated regulation, and a lack of "protective" Th17 and Th22 cells further contribute to epithelial barrier breakdown and MT, thereby potentiating a vicious cycle that ultimately leads to systemic immune activation and its attendant comorbidities. The clinical implications of our in vitro findings are currently speculative and additional in vivo clinical studies that block $\mathrm{MT}^{50-52}$ and those that alter the microbiome composition and bacteria-associated metabolic pathways are required to provide further evidence that interactions between the microbiome and $\mathrm{mDC}$ contribute to intestinal inflammation during HIV-1 infection. These types of studies would also be invaluable in furthering our understanding of the factors contributing to HIV-1-associated mucosal pathogenesis by determining whether the microbiome is altered due to ongoing $\mathrm{mDC}$-mediated mucosal inflammation or whether the increased abundances of pathobionts induce $\mathrm{mDC}$ activation or whether both processes are involved.

\section{METHODS}

Study participants and study design. Twenty-four HIV-1-infected adult subjects and $14 \mathrm{HIV}$-1-seronegative (uninfected) adult control subjects were enrolled in this cross-sectional study at the University of Colorado Anschutz Medical Campus. Efforts were made to enroll control subjects who were matched for age and sex to the HIV-1infected subjects. Clinical characteristics for study subjects are detailed in Table 1. Based on the study entry criteria, HIV-1-infected subjects were cART-naive or had not been on treatment for $>7$ days in the preceding 6 months. Exclusion criteria are extensively described in a previous publication ${ }^{16}$ and detailed in Supplementary Materials. All subjects voluntarily gave written, informed consent. This study was approved by the Colorado Multiple Institutional Review Board at the University of Colorado Anschutz Medical Campus.

Collection, storage, and processing of clinical samples. Collection, storage, and processing of rectal swabs, colon biopsies, and peripheral blood mononuclear cells (PBMC) are detailed in Supplementary Materials.

Enumeration of colonic mucosal HIV-1 viral load. Colonic mucosal HIV-1 viral load was determined as previously described. ${ }^{16}$ To account for variation in the number of CD4 ${ }^{+}$T cells in different samples, HIV RNA copy numbers were normalized per CD4 T cell within each biopsy.

Determination of mucosa-associated bacterial species. Laboratory and analytic methods used to profile the intestinal microbiomes of study participants were described previously. ${ }^{16}$ Species-level taxonomic classification of $16 \mathrm{~S}$ rRNA sequence data sets was obtained via BLAST $^{53}$ of subject sequences against a database built from Silva ${ }^{23}$ bacterial sequences marked as type strains, cultivars, or genomes. A species name was assigned when a sequence overlapped the Silva database sequence by at least $95 \%$ sequence length with at least $99 \%$ sequence identity and the taxonomy of the database hit matched the taxonomy returned by SINA $^{54}$ as determined previously. ${ }^{16}$

Plasma LTA, LPS, and SCD14 measurements. Serum LTA levels were assessed using a custom enzyme-linked immunosorbent assay. ${ }^{16}$ LPS levels were measured in EDTA plasma samples using the Limulus Amebocyte Lysate assay (Lonza, Switzerland) following the manufacturer's protocol as previously detailed. ${ }^{16}$ sCD14 levels were measured in heparin plasma using a commercially available enzyme-linked immunosorbent assay (R\&D Systems, Minneapolis, MN). ${ }^{16}$

Mucosal and plasma cytokine measurements. A Custom Q-plex Array (Quansys Biosciences, Logan, UT) was used to measure mucosal cytokine levels in culture supernatants, and measurement of plasma cytokine levels were performed using the Human Cytokine High Sensitivity Screen as detailed in Supplementary Materials. Levels of plasma IL-6 were evaluated in EDTA plasma samples using a commercially available enzyme-linked immunosorbent assay (R\&D Systems). ${ }^{16}$

Histological staining and analysis of colonic biopsies. Assessment of microbial product levels, CD11 ${ }^{+}$mDCs, and Ham56 ${ }^{+}$macrophages in colonic LP is detailed extensively in Supplementary Materials. Using the Zeiss Zen Software (Zeiss, Jena, Germany), the total area and the area that stained with LTA/LPS was calculated within the LP. To analyze whether microbial products preferentially associated with mDCs or macrophages, the total number of mDCs and macrophages that either did or did not associate with microbial products (LTA/LPS) were enumerated per square millimeter of LP using the Image J Software (NIH, Bethesda, MD)

Assessment of mononuclear infiltration is detailed in Supplementary Materials. Evaluation was performed by a gastrointestinal pathologist who was blinded to the HIV-1 status of each patient. The degree of mononuclear infiltration was quantified on a scale of $0=$ not present, minimal $=0.5$, mild $=1$, moderate $=2$, and severe $=3$.

In vitro stimulations. In vitro mitogenic stimulation of single-cell colon biopsy preparations: Evaluation of frequencies of colonic CD4 T cells capable of producing IFN- $\gamma$ (Th1), IL-17 (Th17) or IL-22 (Th22) and frequencies of IFN- $\gamma$-producing CD8 T cells from isolated colon cells following mitogenic stimulation are detailed in Supplementary Materials.

In vitro exposure of LPMC to commensal bacteria: Cytokine responses by $\mathrm{LPCD} \mathrm{Cc}^{+} \mathrm{mDC}$ to $P$. copri, $P$. stercorea, and $R$. bromii were assessed utilizing an ex vivo tissue culture model consisting of isolated colon LPMC from normal tissue $e^{18,19,49,55}$ and is detailed in Supplementary Materials.

Commensal bacteria stocks. Expansion of P. copri (DSM\# 18205, DSMZ, Braunschweig, Germany), P. stercorea (DSM\# 18206), and R. bromii (ATCC\# 27255, ATCC, Manassas, VA) was performed at $37^{\circ} \mathrm{C}$ under anaerobic conditions per the manufacturer's protocols as described in Supplementary Materials.

Surface and intracellular flow cytometry staining assays, acquisition, and analysis. DC and T-cell frequencies, activation, and cytokine production from colon cells isolated from biopsies and from PBMC: Multi-color flow cytometry protocols to evaluate colon and blood DC and T-cell frequencies and activation status and to determine T-cell cytokine frequencies are detailed in Supplementary Materials. For enumeration of colonic DC and T-cell frequencies, the percentage of DCs or T cells within viable, $\mathrm{CD} 45^{+}$cells was converted to an absolute number per $\mathrm{g}$ based on the frequency within viable, $\mathrm{CD} 45^{+}$cells, initial cell counts, and biopsy weights. Similarly, the percentage of activated colon CD4 and CD8 T cells as well as the percentage of cytokine ${ }^{+}$CD4 and CD8 T cells were also converted to a total number per $\mathrm{g}$ of mucosal tissue.

Phenotypic and functional characterization of $\mathrm{mDC}$ subsets in LPMC from normal colon tissue: Multi-color flow cytometry staining protocols used to characterize $\mathrm{LP} \mathrm{CD1c}{ }^{+} \mathrm{mDCs}$ and $\mathrm{CD} 1 \mathrm{c}^{\text {neg }} \mathrm{mDCs}$ and enumerate cytokine ${ }^{+} \mathrm{CD} \mathrm{c}^{+} \mathrm{mDC}$ following in vitro stimulation of LPMC in normal colon tissue are detailed in Supplementary Materials.

Flow cytometry acquisition: All flow cytometry data were acquired on an LSRII Flow Cytometer (BD Biosciences, San Jose, CA). Routine quality control using the Cytometer Setup and Tracking feature within the BD FACSDiva software version 6.1.2 (BD Biosciences) was performed daily as previously detailed. ${ }^{19}$ 
Statistical analysis. Non-parametric statistics were performed with no adjustments for multiple comparisons due to the exploratory nature of this study. Analysis and graphing were performed using GraphPad Prism Version 6 for Windows (GraphPad Software, San Diego, CA). Comparisons between independent groups were made using Mann-Whitney test and Friedman test with multiple Dunn comparison test for matched paired comparisons across multiple groups. To determine the differences between groups of matched paired data, Wilcoxon's matched-pairs signed-rank test was performed. Correlations between variables were assessed using Spearman's test. Fisher's exact tests and Chi-squared tests were used for comparisons of categorical data. A $P$-value of $<0.05$ was considered significant.

SUPPLEMENTARY MATERIAL is linked to the online version of the paper at http://www.nature.com/mi

\section{ACKNOWLEDGMENTS}

We wish to express our sincere gratitude to all the study participants as well as the physicians and staff at the University of Colorado Infectious Disease Group Practice Clinic. We thank the staff at the Clinical and Translational Research Center (CTRC) and the University Hospital endoscopy clinic for their assistance with our clinical study. We also acknowledge the staff at University of Colorado Hospital CTRC Core Lab for performing the IL-6 assay and the San Diego CFAR Translational Virology Core for performing viral RNA measurements on colon tissue. This study was supported by the National Institutes of Health Grants RO1 DK088663, RO1 Al108404, Al36214 and, in part, by NIH/NCATS Colorado CTSI Grant Number UL1 TR000154.

\section{DISCLOSURE}

The authors declared no conflict of interest.

(c) 2016 Society for Mucosal Immunology

\section{REFERENCES}

1. Hunt, P.W. HIV and inflammation: mechanisms and consequences. Curr. HIVIAIDS Rep. 9, 139-147 (2012).

2. Deeks, S.G. et al. Immune activation set point during early HIV infection predicts subsequent CD4 + T-cell changes independent of viral load. Blood 104, 942-947 (2004)

3. Giorgi, J.V. et al. Shorter survival in advanced human immunodeficiency virus type 1 infection is more closely associated with $T$ lymphocyte activation than with plasma virus burden or virus chemokine coreceptor usage. J. Infect. Dis. 179, 859-870 (1999).

4. Giorgi, J.V. et al. Elevated levels of CD38 + CD8 + T cells in HIV infection add to the prognostic value of low CD4 + Tcell levels: results of 6 years of follow-up. The Los Angeles Center, Multicenter AIDS Cohort Study. J. Acquir. Immune Defic. Syndr. 6, 904-912 (1993).

5. Marchetti, G., Tincati, C. \& Silvestri, G. Microbial translocation in the pathogenesis of HIV infection and AIDS. Clin. Microbiol. Rev. 26, 2-18 (2013).

6. Brenchley, J.M. et al. Microbial translocation is a cause of systemic immune activation in chronic HIV infection. Nat. Med. 12, 1365-1371 (2006).

7. Marchetti, G. et al. Microbial translocation predicts disease progression of HIV-infected antiretroviral-naive patients with high CD4 + cell count. AIDS 25, 1385-1394 (2011)

8. Hunt, P.W. et al. Gut epithelial barrier dysfunction and innate immune activation predict mortality in treated HIV infection. J. Infect. Dis. 210, 1228-1238 (2014).

9. Sandler, N.G. et al. Plasma levels of soluble CD14 independently predict mortality in HIV infection. J. Infect. Dis. 203, 780-790 (2011).

10. George, M.D. \& Asmuth, D.M. Mucosal immunity in HIV infection: what can be done to restore gastrointestinal-associated lymphoid tissue function? Curr. Opin. Infect. Dis. 27, 275-281 (2014).

11. Hayes, T.L. et al. Impact of highly active antiretroviral therapy initiation on CD4(+) T-cell repopulation in duodenal and rectal mucosa. AIDS 27, 867-877 (2013).
12. Kok, A. et al. Early initiation of combined antiretroviral therapy preserves immune function in the gut of HIV-infected patients. Mucosal Immunol. 8, 127-140 (2015).

13. Schneider, T. et al. Abnormalities in subset distribution, activation, and differentiation of $\mathrm{T}$ cells isolated from large intestine biopsies in HIV infection. The Berlin Diarrhoea/Wasting Syndrome Study Group. Clin. Exp. Immunol. 95, 430-435 (1994).

14. McGowan, I. et al. Increased HIV-1 mucosal replication is associated with generalized mucosal cytokine activation. J. Acquir. Immune Defic. Syndr. 37, 1228-1236 (2004).

15. Mutlu, E.A. et al. A compositional look at the human gastrointestinal microbiome and immune activation parameters in HIV infected subjects. PLoS Pathogens 10, e1003829 (2014).

16. Dillon, S.M. et al. An altered intestinal mucosal microbiome in HIV-1 infection is associated with mucosal and systemic immune activation and endotoxemia. Mucosal Immunol. 7, 983-994 (2014).

17. Chirdo, F.G., Millington, O.R., Beacock-Sharp, H. \& Mowat, A.M. Immunomodulatory dendritic cells in intestinal lamina propria. Eur. J. Immunol. 35, 1831-1840 (2005).

18. Dillon, S.M. et al. Human intestinal lamina propria CD1C+ dendritic cells display an activated phenotype at steady state and produce IL-23 in response to TLR7/8 stimulation. J. Immunol. 184, 6612-6621 (2010).

19. Dillon, S.M. et al. HIV-1 infection of human intestinal lamina propria CD4 + T cells in vitro is enhanced by exposure to commensal Escherichia coli. J. Immunol. 189, 885-896 (2012).

20. Allers, K. et al. Macrophages accumulate in the gut mucosa of untreated HIV-infected patients. J. Infect. Dis. 209, 739-748 (2014).

21. Dillon, S.M. et al. Plasmacytoid and myeloid dendritic cells with a partial activation phenotype accumulate in lymphoid tissue during asymptomatic chronic HIV-1 infection. J. Acquir. Immune Defic. Syndr. 48, 1-12 (2008).

22. O'Brien, M., Manches, O. \& Bhardwaj, N. Plasmacytoid dendritic cells in HIV infection. Adv. Exp. Med. Biol. 762, 71-107 (2013).

23. Quast, C. etal. The SILVA ribosomal RNA gene database project: improved data processing and web-based tools. Nucleic Acids Res. 41, D590-D596 (2013).

24. Klatt, N.R. et al. Loss of mucosal CD103 + DCs and IL-17 + and IL-22 + lymphocytes is associated with mucosal damage in SIV infection. Mucosal Immunol. 5, 646-657 (2012).

25. Kwa, S. et al. Plasmacytoid dendritic cells are recruited to the colorectum and contribute to immune activation during pathogenic SIV infection in rhesus macaques. Blood 118, 2763-2773 (2011).

26. Reeves, R.K. et al. SIV infection induces accumulation of plasmacytoid dendritic cells in the gut mucosa. J. Infect. Dis. 206, 1462-1468 (2012).

27. Wijewardana, V. et al. Kinetics of myeloid dendritic cell trafficking and activation: impact on progressive, nonprogressive and controlled SIV infections. PLoS Pathogens 9, e1003600 (2013).

28. Kumar, P.S. et al. New bacterial species associated with chronic periodontitis. J Dent. Res. 82, 338-344 (2003).

29. Lucke, K., Miehlke, S., Jacobs, E. \& Schuppler, M. Prevalence of Bacteroides and Prevotella spp. in ulcerative colitis. J. Med. Microbiol. 55, 617-624 (2006).

30. Scher, J.U. et al. Expansion of intestinal Prevotella copri correlates with enhanced susceptibility to arthritis. eLife 2, e01202 (2013).

31. Aerts-Toegaert, C. et al. CD83 expression on dendritic cells and T cells: correlation with effective immune responses. Eur. J. Immunol. 37, 686-695 (2007).

32. Prechtel, A.T., Turza, N.M., Theodoridis, A.A. \& Steinkasserer, A. CD83 knockdown in monocyte-derived dendritic cells by small interfering RNA leads to a diminished T cell stimulation. J. Immunol. 178, 5454-5464 (2007).

33. Arrode, G., Boccaccio, C., Abastado, J.P. \& Davrinche, C. Crosspresentation of human cytomegalovirus pp65 (UL83) to CD8 + T cells is regulated by virus-induced, soluble-mediator-dependent maturation of dendritic cells. J. Virol. 76, 142-150 (2002).

34. Kruse, M. et al. Mature dendritic cells infected with herpes simplex virus type 1 exhibit inhibited T-cell stimulatory capacity. J. Virol. 74, 7127-7136 (2000). 
35. Silva, M.A. et al. Dendritic cells and toll-like receptors 2 and 4 in the ileum of Crohn's disease patients. Dig. Dis. Sci. 53, 1917-1928 (2008).

36. Ahern, P.P., Izcue, A., Maloy, K.J. \& Powrie, F. The interleukin-23 axis in intestinal inflammation. Immunol. Rev. 226, 147-159 (2008).

37. Santarlasci, V., Cosmi, L., Maggi, L., Liotta, F. \& Annunziato, F. IL-1 and T helper immune responses. Front. Immunol. 4, 182 (2013).

38. Brenchley, J.M. et al. CD4 + T cell depletion during all stages of HIV disease occurs predominantly in the gastrointestinal tract. J. Exp. Med. 200, 749-759 (2004).

39. Maynard, C.L. \& Weaver, C.T. Diversity in the contribution of interleukin-10 to T-cell-mediated immune regulation. Immunol. Rev. 226, 219-233 (2008).

40. Norris, P.J. et al. Elevations in IL-10, TNF-alpha, and IFN-gamma from the earliest point of HIV Type 1 infection. AIDS Res. Hum. Retroviruses 22, 757-762 (2006).

41. Stacey, A.R. et al. Induction of a striking systemic cytokine cascade prior to peak viremia in acute human immunodeficiency virus type 1 infection, in contrast to more modest and delayed responses in acute hepatitis B and C virus infections. J. Virol. 83, 3719-3733 (2009).

42. Lauw, F.N. et al. Proinflammatory effects of IL-10 during human endotoxemia. J. Immunol. 165, 2783-2789 (2000).

43. Manuzak, J., Dillon, S. \& Wilson, C. Differential interleukin-10 (IL-10) and IL-23 production by human blood monocytes and dendritic cells in response to commensal enteric bacteria. Clin. Vaccine Immunol. 19, 1207-1217 (2012).

44. Estes, J.D. et al. Damaged intestinal epithelial integrity linked to microbial translocation in pathogenic simian immunodeficiency virus infections. PLoS Pathogens 6, e1001052 (2010).

45. Smythies, L.E. et al. Human intestinal macrophages display profound inflammatory anergy despite avid phagocytic and bacteriocidal activity. J. Clin. Invest. 115, 66-75 (2005).
46. Fonteneau, J.F. et al. Human immunodeficiency virus type 1 activates plasmacytoid dendritic cells and concomitantly induces the bystander maturation of myeloid dendritic cells. J. Virol. 78, 5223-5232 (2004).

47. Lehmann, C. et al. Longitudinal analysis of distribution and function of plasmacytoid dendritic cells in peripheral blood and gut mucosa of HIV infected patients. J. Infect. Dis. 209, 940-949 (2014).

48. Li, H., Gillis, J., Johnson, R.P. \& Reeves, R.K. Multi-functional plasmacytoid dendritic cells redistribute to gut tissues during simian immunodeficiency virus infection. Immunology 140, 244-249 (2013).

49. Steele, A.K. et al. Microbial exposure alters HIV-1-induced mucosal CD4 + T cell death pathways ex vivo. Retrovirology 11, 14 (2014).

50. Kristoff, J. et al. Early microbial translocation blockade reduces SIV-mediated inflammation and viral replication. J. Clin. Invest. 124, 2802-2806 (2014).

51. Sandler, N.G. et al. Sevelamer does not decrease lipopolysaccharide or soluble CD14 levels but decreases soluble tissue factor, low-density lipoprotein (LDL) cholesterol, and oxidized LDL cholesterol levels in individuals with untreated HIV infection. J. Infect. Dis. 210, 1549-1554 (2014).

52. Tenorio, A.R. et al. Rifaximin has a marginal impact on microbial translocation, T-cell activation and inflammation in HIV-positive immune non-responders to antiretroviral therapy—ACTG A5286. J. Infect. Dis. 211, 780-790 (2015).

53. Altschul, S.F., Gish, W., Miller, W., Myers, E.W. \& Lipman, D.J. Basic local alignment search tool. J. Mol. Biol. 215, 403-410 (1990).

54. Pruesse, E., Peplies, J. \& Glockner, F.O. SINA: accurate high-throughput multiple sequence alignment of ribosomal RNA genes. Bioinformatics $\mathbf{2 8}$, 1823-1829 (2012).

55. Howe, R. et al. Evidence for dendritic cell-dependent CD4(+) Thelper-1 type responses to commensal bacteria in normal human intestinal lamina propria. Clin. Immunol. 131, 317-332 (2009). 